\title{
Localizing virtual structure sheaves for almost perfect obstruction theories
}

\author{
Young-Hoon Kiem ${ }^{1}$ and Michail Savvas ${ }^{2}$ \\ ${ }^{1}$ Department of Mathematical Sciences and Research Institute of Mathematics, Seoul National University, Seoul 08826, Korea; \\ E-mail:kiem@snu.ac.kr. \\ ${ }^{2}$ Department of Mathematics, University of California, San Diego, La Jolla CA 92093, USA; E-mail: msavvas@ucsd.edu.
}

Received: 28 July 2020; Revised: 13 September 2020; Accepted: 12 November 2020

2020 Mathematics Subject Classification: Primary - 14N35; Secondary - 14C35, 14D23

\begin{abstract}
Almost perfect obstruction theories were introduced in an earlier paper by the authors as the appropriate notion in order to define virtual structure sheaves and $K$-theoretic invariants for many moduli stacks of interest, including $K$-theoretic Donaldson-Thomas invariants of sheaves and complexes on Calabi-Yau threefolds. The construction of virtual structure sheaves is based on the $K$-theory and Gysin maps of sheaf stacks.

In this paper, we generalize the virtual torus localization and cosection localization formulas and their combination to the setting of almost perfect obstruction theory. To this end, we further investigate the $K$-theory of sheaf stacks and its functoriality properties. As applications of the localization formulas, we establish a $K$-theoretic wallcrossing formula for simple $\mathbb{C}^{*}$-wall crossings and define $K$-theoretic invariants refining the Jiang-Thomas virtual signed Euler characteristics.
\end{abstract}

\section{Contents}

1 Introduction

$2 K$-theory on sheaf stacks, almost perfect obstruction theory and the virtual structure sheaf 4

2.1 Sheaf stacks, local charts and common roofs . . . . . . . . . . . . . . . . 4

2.2 Coherent sheaves on a sheaf stack $\mathcal{F} \ldots \ldots \ldots \ldots$

$2.3 K$-theoretic Gysin and pullback maps . . . . . . . . . . . . . . . 6

2.4 Almost perfect obstruction theory and virtual structure sheaf $\ldots \ldots \ldots$. . . . . . 7

3 Functorial behavior of coherent sheaves on sheaf stacks $\quad 8$

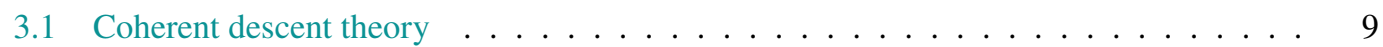

3.2 Pullbacks of coherent sheaves on sheaf stacks . . . . . . . . . . . . 11

3.3 Pushforwards of coherent sheaves on sheaf stacks . . . . . . . . . . . . . 13

4 Cosection localization $\quad 14$

4.1 Cosection localization for perfect obstruction theory . . . . . . . . . . . . . . . . . 14

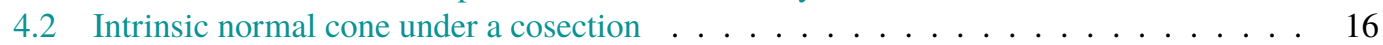

4.3 Cosection localized Gysin maps . . . . . . . . . . . . . . . . . . 17

4.4 Cosection localized virtual structure sheaf for almost perfect obstruction theory . . . 18

5 Virtual torus localization $\quad 19$

$5.1 T$-equivariant almost perfect obstruction theory . . . . . . . . . . . 19

$5.2 T$-equivariant almost perfect obstruction theory on the fixed locus . . . . . . . 20

(C) The Author(s), 2020. Published by Cambridge University Press. This is an Open Access article, distributed under the terms of the Creative Commons Attribution licence (http://creativecommons.org/licenses/by/4.0/), which permits unrestricted re-use, distribution, and reproduction in any medium, provided the original work is properly cited. 
5.3 An auxiliary almost perfect obstruction theory on the fixed locus . . . . . . . . . 22

5.4 Refined intersection with the fixed locus . . . . . . . . . . . . . . 22

5.5 Virtual torus localization formula . . . . . . . . . . . . . . . 25

6 Torus localization of cosection localized virtual structure sheaf $\quad 28$

7 Applications $\quad 29$

$7.1 \quad K$-theoretic simple $\mathbb{C}^{*}$-wall crossing . . . . . . . . . . . . . . . . . . 29

7.2 Dual obstruction cone . . . . . . . . . . . . . . . . . . 30

A Deformation invariance of cosection localized virtual structure sheaf 32

\section{Introduction}

Enumerative geometry is the study of counts of geometric objects subject to a set of given conditions. More often than not, the moduli stacks parameterizing the objects of interest are highly singular, have many components of dimension different from the expected dimension and do not behave well under deformation.

To address these issues, Li-Tian [20] and Behrend-Fantechi [4] developed the theory of virtual fundamental cycles, which have been instrumental in defining and investigating several algebro-geometric enumerative invariants of great importance, such as Gromov-Witten [2], Donaldson-Thomas [29] and Pandharipande-Thomas [25] invariants, and are still one of the major components in modern enumerative geometry.

Any Deligne-Mumford stack $X$ is equipped with its intrinsic normal cone $\mathcal{C}_{X}$, which locally for an étale map $U \rightarrow X$ and a closed embedding $U \hookrightarrow V$ into a smooth scheme $V$ is the quotient stack $\left[C_{U / V} /\left.T_{V}\right|_{U}\right]$ (see also [4]). A perfect obstruction theory $\phi: E \rightarrow \mathbb{L}_{X}$ gives an embedding of $\mathcal{C}_{X}$ into the vector bundle stack $\mathcal{E}_{X}=h^{1} / h^{0}\left(E^{\vee}\right)$, and the virtual fundamental cycle is defined as the intersection of the zero section $0_{\mathcal{E}_{X}}$ with $\mathcal{C}_{X}$

$$
[X]^{\text {vir }}:=0_{\mathcal{E}_{X}}^{!}\left[\mathcal{C}_{X}\right] \in A_{\text {v.dim. }}(X) .
$$

Recently, a lot of interest has been generated toward refinements of enumerative invariants that go beyond numbers or the intersection theory of cycles. Motivated by theoretical physics and geometric representation theory, it is in particular desirable to obtain such a refinement in $K$-theory (see also, for example, $[23,24])$.

When the moduli stack $X$ admits a perfect obstruction theory with a global presentation $E=\left[E^{-1} \rightarrow\right.$ $\left.E^{0}\right]$, where $E^{-1}, E^{0}$ are locally free sheaves on $X$, one can define the virtual structure sheaf as

$$
\left[\mathcal{G}_{X}^{\mathrm{vir}}\right]:=0_{E_{1}}^{!}\left[\mathcal{G}_{C_{1}}\right] \in K_{0}(X)
$$

where $E_{1}=\left(E^{-1}\right)^{\vee}$ and $C_{1}=\mathcal{C}_{X} \times_{\mathcal{E}_{X}} E_{1}$.

However, there are many moduli stacks of interest that do not admit perfect obstruction theories, including moduli of simple complexes $[9,21]$ and desingularizations of stacks of semistable sheaves and perfect complexes on Calabi-Yau threefolds [15, 28].

To resolve this, in our previous paper [16], we introduced a relaxed version of perfect obstruction theories, called almost perfect obstruction theories, which arise in the above moduli stacks. An almost perfect obstruction theory has an obstruction sheaf $\mathcal{O} b_{X}$, which is the analogue of the sheaf $h^{1}\left(E^{\vee}\right)$, and induces an embedding of the coarse moduli sheaf $c_{X}$ of $\mathcal{C}_{X}$ into $\mathcal{O} b_{X}$, enabling us to define the virtual structure sheaf of $X$ as

$$
\left[\bigcirc_{X}^{\mathrm{vir}}\right]:=0_{\mathcal{O} b_{X}}^{!}\left[\bigcirc_{\mathfrak{c}_{X}}\right] \in K_{0}(X) .
$$

Several techniques have been developed to handle virtual fundamental cycles and virtual structure sheaves arising from perfect obstruction theories on Deligne-Mumford stacks, such as the virtual torus localization of Graber-Pandharipande [7, 26], the cosection localization of Kiem-Li [12, 14], virtual 
pullback [22, 26] and wall-crossing formulas [13]. Often, combining these (see also, for example, [5]) can be quite effective.

The aim of the present paper is to generalize the virtual torus localization and cosection localization formulas of virtual structure sheaves and their combination to the setting of almost perfect obstruction theory.

Roughly speaking, an almost perfect obstruction theory $\phi$ on a Deligne-Mumford stack $X$ consists of perfect obstruction theories

$$
\phi_{\alpha}: E_{\alpha} \longrightarrow \mathbb{L}_{U_{\alpha}}
$$

on an étale cover $\left\{U_{\alpha} \rightarrow X\right\}$ of $X$ satisfying appropriate combatibility conditions (see also Definition 2.10), which ensure that we have an obstruction sheaf $\mathcal{F}=\mathcal{O} b_{X}$ and an embedding of the coarse intrinsic normal cone $\mathfrak{c}_{X}$ into $\mathcal{F}$, so that the virtual structure sheaf $\left[\mathcal{O}_{X}^{\text {vir }}\right]$ can be defined as in (1.1) above.

The definition of $\left[\mathcal{O}_{X}^{\mathrm{vir}}\right]$ is based crucially on the development of a $K$-theory group $K_{0}(\mathcal{F})$ of coherent sheaves on the sheaf stack $\mathcal{F}$, so that $\left[\sigma_{\mathfrak{c}_{X}}\right] \in \operatorname{Coh}(\mathcal{F})$, and the construction of the Gysin map $0 !: K_{0}(\mathcal{F}) \rightarrow K_{0}(X)$ in [16].

In this paper, we investigate the $K$-theory of sheaf stacks in more detail and establish an appropriate version of descent theory for the category $\operatorname{Coh}(\mathcal{F})$ of coherent sheaves on $\mathcal{F}$ and several functorial behaviors for $\operatorname{Coh}(\mathcal{F})$ and $K_{0}(\mathcal{F})$ and their properties. These include:

1. Pullback functors for surjective homomorphisms $\mathcal{G} \rightarrow \mathcal{F}$

2. Pullback and pushforward functors for injective homomorphisms $\mathcal{G} \rightarrow \mathcal{F}$ with locally free quotient $L=\mathcal{F} / \mathcal{G}$

3. Pullback and pushforward functors for morphisms $\tau: Y \rightarrow X$ of the base

With these well developed, after appropriate modifications, the standard arguments proving the virtual torus localization formula, cosection localization formula and their combination work in our setting.

Thus, when $X$ admits a $T=\mathbb{C}^{*}$-action, and the cover $\left\{U_{\alpha} \rightarrow X\right\}$ and obstruction theories $\phi_{\alpha}$ are $T$-equivariant, we prove that (Theorem 5.15)

$$
\left[\circlearrowleft_{X}^{\mathrm{vir}}\right]=\iota_{*} \frac{\left[\circlearrowleft_{F}^{\mathrm{vir}}\right]}{e\left(N^{\mathrm{vir}}\right)} \in K_{0}^{T}(X) \otimes_{\mathbb{Q}\left[t, t^{-1}\right]} \mathbb{Q}(t)
$$

where $\iota: F \rightarrow X$ is the inclusion of the $T$-fixed locus, which admits an induced almost perfect obstruction theory, and we assume that the virtual normal bundle $N^{\text {vir }}$ of $F$ has a global resolution $\left[N_{0} \rightarrow N_{1}\right]$ by locally free sheaves.

Moreover, if there is a cosection $\sigma: \mathcal{O} b_{X} \rightarrow \mathcal{O}_{X}$ with vanishing locus $X(\sigma)$, we prove (Definition 4.5, Proposition 4.6) that the virtual structure sheaf $\left[\mathcal{O}_{X}^{\mathrm{vir}}\right]$ localizes canonically to an element $\left[\mathcal{O}_{X, \mathrm{loc}}^{\mathrm{vir}}\right] \in$ $K_{0}(X(\sigma))$.

In the presence of a $T$-action such that the almost perfect obstruction theory is $T$-equivariant and a $T$-invariant cosection $\sigma$, we show that the virtual torus localization formula holds for the cosection localized virtual structure sheaves of $X$ and $F$ (Theorem 6.1).

An immediate application of the virtual torus localization formula is a $K$-theoretic wall-crossing formula for simple $\mathbb{C}^{*}$-wall crossings (Theorem 7.2).

Another application is a $K$-theoretic refinement of the Jiang-Thomas theory of virtual signed Euler characteristics [10]. If $X$ is a Deligne-Mumford stack with a perfect obstruction theory with obstruction sheaf $\mathcal{F}=\mathcal{O} b_{X}$, then we show (Theorem 7.4) that the dual obstruction cone $N=\operatorname{Spec}_{X}(\operatorname{Sym} \mathcal{F})$ admits a (symmetric) almost perfect obstruction theory with obstruction sheaf $\mathcal{O} b_{N}=\Omega_{N}$. This is $T$-equivariant for the natural $T$-action with fixed locus $\iota: X \rightarrow N$. Additionally, there is a cosection 
$\sigma: \mathcal{O} b_{N} \rightarrow \mathcal{O}_{N}$. When $X$ is proper, we obtain (Theorem 7.6) the $K$-theoretic invariants

$$
\chi\left(\left[\mathcal{G}_{N, \text { loc }}^{\mathrm{vir}}\right]\right) \in \mathbb{Q}, \quad \chi_{t}\left(\frac{\left[\mathcal{G}_{X}^{\mathrm{vir}}\right]}{e\left(E^{\vee}\right)}\right) \in \mathbb{Q}(t)
$$

refining the Jiang-Thomas virtual signed Euler characteristics.

\section{Layout of the paper}

Section 2 collects necessary background on the $K$-theory of sheaf stacks, almost perfect obstruction theories and virtual structure sheaves that we need from [16]. In Section 3, we study the descent theory and functoriality properties of coherent sheaves on sheaf stacks, which are then used throughout the rest of the paper. Section 4 treats the cosection localization formula, Section 5 treats the virtual torus localization formula and Section 6 their combination. In Section 7, we apply these formulas to prove the $K$-theoretic wall-crossing formula for simple $\mathbb{C}^{*}$-wall crossings and construct a $K$-theoretic refinement of the Jiang-Thomas theory of virtual signed Euler characteristics. Finally, in the Appendix, we give the proof of the deformation invariance of the cosection localized virtual structure sheaf.

\section{Notation and conventions}

Everything in this paper is over the field $\mathbb{C}$ of complex numbers. All stacks are of finite type, and Deligne-Mumford stacks are separated.

If $E$ is a locally free sheaf on a Deligne-Mumford stack $X$, we will use the term vector bundle to refer to its total space. If $\mathcal{F}$ is a coherent sheaf on a Deligne-Mumford stack $X$, we will use the same letter to refer to the associated sheaf stack.

For a morphism $f: Y \rightarrow X$ of stacks and a coherent sheaf $\mathcal{F}$ on $X$, its pullback $f^{*} \mathcal{F}$ is sometimes denoted by $\left.\mathcal{F}\right|_{Y}$ when the map $f$ is clear from the context. The bounded derived category of coherent sheaves on a stack $X$ is denoted by $D(X)$, and $\mathbb{L}_{X / S}=L_{X / S}^{\geq-1}$ denotes the truncated cotangent complex for a morphism $X \rightarrow S$. T typically denotes the torus $\mathbb{C}^{*}$.

\section{2. $K$-theory on sheaf stacks, almost perfect obstruction theory and the virtual structure sheaf}

In [16], we introduced the notion of coherent sheaves on a sheaf stack $\mathcal{F}$, defined the $K$-theory of $\mathcal{F}$ and constructed a Gysin map $0 !_{\mathcal{F}}$ of $\mathcal{F}$, which enabled us to construct the virtual structure sheaf $\left[\bigcirc_{X}^{\mathrm{vir}}\right] \in K_{0}(X)$ for a Deligne-Mumford stack $X$ equipped with an almost perfect obstruction theory.

In this preliminary section, we collect necessary ingredients from [16].

\subsection{Sheaf stacks, local charts and common roofs}

In what follows, $X$ will denote a Deligne-Mumford stack and $\mathcal{F}$ a coherent sheaf on $X$.

Definition 2.1. (Sheaf stack) The sheaf stack associated with $\mathcal{F}$ is the stack that to every morphism $\rho: W \rightarrow X$ from a scheme $W$ associates the set $\Gamma\left(W, \rho^{*} \mathcal{F}\right)$.

By abuse of notation, we denote by $\mathcal{F}$ the sheaf stack associated with a coherent sheaf $\mathcal{F}$ on $X$. A sheaf stack is not algebraic in general, and we need an appropriate notion of local charts for geometric constructions.

Definition 2.2. (Local chart) A local chart $Q=\left(U, \rho, E, r_{E}\right)$ for the sheaf stack $\mathcal{F}$ consists of

1. An étale morphism $\rho: U \rightarrow X$ from a scheme $U$, and

2. A surjective homomorphism $r_{E}: E \rightarrow \rho^{*} \mathcal{F}=\left.\mathcal{F}\right|_{U}$ of coherent sheaves on $U$ from a locally free sheaf $E$ on $U$. 
We will call $U$ the base of the chart $Q$. If $U$ is affine and $E$ is free, then the local chart $Q=\left(U, \rho, E, r_{E}\right)$ is called affine.

Definition 2.3. (Morphism between local charts) Let $Q=\left(U, \rho, E, r_{E}\right)$ and $Q^{\prime}=\left(U^{\prime}, \rho^{\prime}, E^{\prime}, r_{E^{\prime}}\right)$ be two local charts for $\mathcal{F}$. A morphism $\gamma: Q \rightarrow Q^{\prime}$ is the pair $\left(\rho_{\gamma}, r_{\gamma}\right)$ of an étale morphism $\rho_{\gamma}: U \rightarrow U^{\prime}$ and a surjection $r_{\gamma}: E \rightarrow \rho_{\gamma}^{*} E^{\prime}$ of locally free sheaves, such that the diagrams
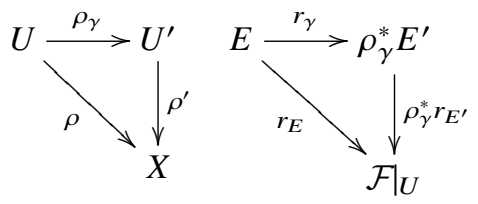

are commutative.

We say that $Q$ is a restriction of $Q^{\prime}$ and write $Q=\left.Q^{\prime}\right|_{U}$ if $E=\rho_{\gamma}^{*} E^{\prime}$ and $r_{\gamma}$ is the identity morphism.

The notion of a common roof enables us compare two local charts on $\mathcal{F}$ with the same base $\rho: U \rightarrow X$.

Definition 2.4. (Common roof) Let $r: \mathcal{G} \rightarrow \mathcal{F}$ and $r^{\prime}: \mathcal{G}^{\prime} \rightarrow \mathcal{F}$ be two surjective homomorphisms of coherent sheaves on a scheme $U$. Their fiber product is defined by

$$
\mathcal{G} \times_{\mathcal{F}} \mathcal{G}^{\prime}:=\operatorname{ker}\left(\mathcal{G} \oplus \mathcal{G}^{\prime} \stackrel{\left(r,-r^{\prime}\right)}{\longrightarrow} \mathcal{F} \oplus \mathcal{F} \stackrel{+}{\rightarrow} \mathcal{F}\right)
$$

and we have a commutative diagram

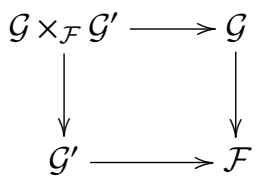

of surjective homomorphisms, which is universal among such diagrams of surjective homomorphisms in the obvious sense.

Given two charts $Q=\left(U, \rho, E, r_{E}\right)$ and $Q^{\prime}=\left(U, \rho, E^{\prime}, r_{E^{\prime}}\right)$ with the same quasi-projective base $U$, we can pick a surjective homomorphism

$$
W \longrightarrow E \times_{\left.\mathcal{F}\right|_{U}} E^{\prime}
$$

from a locally free sheaf $W$. Denoting the induced surjection $\left.W \rightarrow \mathcal{F}\right|_{U}$ by $r_{W}$, we obtain a local chart $\left(U, \rho, W, r_{W}\right)$, which we call a common roof of $Q$ and $Q^{\prime}$.

More generally, given two charts $Q=\left(U, \rho, E, r_{E}\right)$ and $Q^{\prime}=\left(U^{\prime}, \rho^{\prime}, E^{\prime}, r_{E^{\prime}}\right)$ of the sheaf stack $\mathcal{F}$, we let $V=U \times_{X} U^{\prime}$ and have two local charts $\left.Q\right|_{V}$ and $\left.Q^{\prime}\right|_{V}$ with the same base. A common roof of $\left.Q\right|_{V}$ and $\left.Q^{\prime}\right|_{V}$ is called a common roof of $Q$ and $Q^{\prime}$.

\subsection{Coherent sheaves on a sheaf stack $\mathcal{F}$}

A coherent sheaf $\mathcal{A}$ on $\mathcal{F}$ is an assignment to every local chart $Q=\left(U, \rho, E, r_{E}\right)$ of a coherent sheaf $\mathcal{A}_{Q}$ on the scheme $E$ (in the étale topology) such that for every morphism $\gamma: Q \rightarrow Q^{\prime}$ between local charts there exists an isomorphism

$$
r_{\gamma}^{*}\left(\rho_{\gamma}^{*} \mathcal{A}_{Q^{\prime}}\right) \longrightarrow \mathcal{A}_{Q}
$$

that satisfies the usual compatibilities for composition of morphisms. Note that we abusively write $\rho_{\gamma}^{*} \mathcal{A}_{Q^{\prime}}$ for the pullback of $\mathcal{A}_{Q^{\prime}}$ to $\rho_{\gamma}^{*} E^{\prime}$ via the morphism of bundles $\rho_{\gamma}^{*} E^{\prime} \rightarrow E^{\prime}$ induced by $\rho_{\gamma}$. A quasicoherent sheaf on a sheaf stack is defined likewise. 
A homomorphism $f: \mathcal{A} \rightarrow \mathcal{B}$ of (quasi)coherent sheaves on $\mathcal{F}$ is the data of a homomorphism $f_{Q}: \mathcal{A}_{Q} \rightarrow \mathcal{B}_{Q}$ of (quasi)coherent sheaves on $E$ for each local chart $Q=\left(U, \rho, E, r_{E}\right)$ such that for every morphism $\gamma: Q \rightarrow Q^{\prime}$ of local charts, the diagram

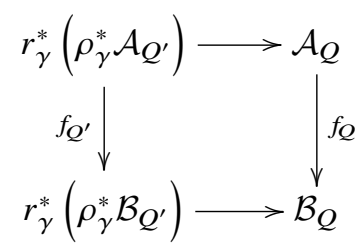

is commutative where the horizontal arrows are (2.2). We say that a homomorphism $f: \mathcal{A} \rightarrow \mathcal{B}$ is an isomorphism if $f_{Q}$ is an isomorphism for each local chart $Q$.

Exact sequences and the $K$-group $K_{0}(\mathcal{F})$ were defined in [16] as follows.

Definition 2.5. (Short exact sequence) Let $\mathcal{A}, \mathcal{B}, \mathcal{C}$ be coherent sheaves on the sheaf stack $\mathcal{F}$. A sequence

$$
0 \longrightarrow \mathcal{A} \longrightarrow \mathcal{B} \longrightarrow \mathcal{C} \longrightarrow 0
$$

of homomorphisms of coherent sheaves on $\mathcal{F}$ is exact if for every local chart $Q=\left(U, \rho, E, r_{E}\right)$ on $\mathcal{F}$ the sequence

$$
0 \longrightarrow \mathcal{A}_{Q} \longrightarrow \mathcal{B}_{Q} \longrightarrow \mathcal{C}_{Q} \longrightarrow 0
$$

is an exact sequence of coherent sheaves on the scheme $E$.

Note that the morphism

$$
E \stackrel{r_{\gamma}}{\longrightarrow} \rho_{\gamma}^{*} E^{\prime}=U \times_{U^{\prime}} E^{\prime} \longrightarrow E^{\prime}
$$

is smooth and hence flat. We can likewise define the kernel and cokernel of a homomorphisms $f: \mathcal{A} \rightarrow \mathcal{B}$ of coherent sheaves on the sheaf stack $\mathcal{F}$. Thus coherent and quasicoherent sheaves on $\mathcal{F}$ form abelian categories

$$
\operatorname{Coh}(\mathcal{F}) \subset \mathrm{QCoh}(\mathcal{F})
$$

Definition 2.6. The $K$-group of coherent sheaves on $\mathcal{F}$ is the group generated by the isomorphism classes $[\mathcal{A}]$ of coherent sheaves $\mathcal{A}$ on $\mathcal{F}$, with relations generated by $[\mathcal{B}]=[\mathcal{A}]+[\mathcal{C}]$ for every short exact sequence

$$
0 \longrightarrow \mathcal{A} \longrightarrow \mathcal{B} \longrightarrow \mathcal{C} \longrightarrow 0
$$

In other words, $K_{0}(\mathcal{F})$ is the Grothendieck group of the abelian category $\operatorname{Coh}(\mathcal{F})$.

If $\mathcal{F}=E$ is locally free, so that $\mathcal{F}$ is an algebraic stack, then the above definitions recover the standard notions of short exact sequences and $K_{0}(\mathcal{F})$ for the vector bundle $E$.

\subsection{K-theoretic Gysin and pullback maps}

Let $Q=\left(U, \rho, E, r_{E}\right)$ be a local chart for the sheaf stack $\mathcal{F}$, and denote the vector bundle projection map $E \rightarrow U$ by $\pi_{E}$. The tautological section of the pullback $\pi_{E}^{*} E$ induces an associated Koszul complex

$$
\mathcal{K}(E):=\wedge^{\bullet} \pi_{E}^{*} E^{\vee}
$$

that resolves the structure sheaf $\sigma_{U}$ of the zero section of $\pi_{E}$. 
Definition 2.7. For any local chart $Q=\left(U, \rho, E, r_{E}\right)$ and a coherent sheaf $\mathcal{A}$ on $\mathcal{F}$, the ith Koszul homology sheaf

$$
\mathcal{H}_{Q}^{i}(\mathcal{A}) \in \operatorname{Coh}(U)
$$

of $\mathcal{A}$ with respect to $Q$ is defined as the homology of the complex

$$
\mathcal{K}(E) \otimes_{\mathscr{G}_{E}} \mathcal{A}_{Q}=\wedge^{\bullet} \pi_{E}^{*} E^{\vee} \otimes_{\mathscr{G}_{E}} \mathcal{A}_{Q}
$$

in degree $-i$.

Using common roofs and the standard descent theory for coherent sheaves on algebraic stacks, the following is proven in [16].

Theorem-Definition 2.8. Let $\mathcal{A}$ be a coherent sheaf on a sheaf stack $\mathcal{F}$ over a Deligne-Mumford stack $X$. The coherent sheaves $\mathcal{H}_{Q}^{i}(\mathcal{A}) \in \operatorname{Coh}(U)$ glue canonically to a coherent sheaf $\mathcal{H}_{\mathcal{K}}^{i}(\mathcal{A}) \in \operatorname{Coh}(X)$ on $X$, which is defined to be the ith Koszul homology sheaf of $\mathcal{A}$.

The Koszul homology sheaves of $\mathcal{A}$ were then used to define a $K$-theoretic Gysin map $0_{\mathcal{F}}: K_{0}(\mathcal{F}) \rightarrow$ $K_{0}(X)$.

Definition 2.9. ( $K$-theoretic Gysin map) The $K$-theoretic Gysin map is defined by the formula

$$
0_{\mathcal{F}}^{!}: K_{0}(\mathcal{F}) \longrightarrow K_{0}(X), \quad 0_{\mathcal{F}}^{!}[\mathcal{A}]=\sum_{i \geq 0}(-1)^{i}\left[\mathcal{H}_{\mathcal{K}}^{i}(\mathcal{A})\right] \in K_{0}(X)
$$

where $\mathcal{A}$ is a coherent sheaf on $\mathcal{F}$.

\subsection{Almost perfect obstruction theory and virtual structure sheaf}

A perfect obstruction theory on a morphism $X \rightarrow S$, where $X$ is a scheme and $S$ a smooth Artin stack, is a morphism

$$
\phi: E \longrightarrow \mathbb{L}_{X / S}
$$

in $D(X)$, where $E$ is a perfect complex of amplitude [-1,0], satisfying that $h^{-1}(\phi)$ is surjective and $h^{0}(\phi)$ is an isomorphism.

We refer to the sheaf $\mathcal{O} b_{X}:=h^{1}\left(E^{\vee}\right)$ as the obstruction sheaf associated with the perfect obstruction theory $\phi$. Then $\phi$ induces a Cartesian diagram

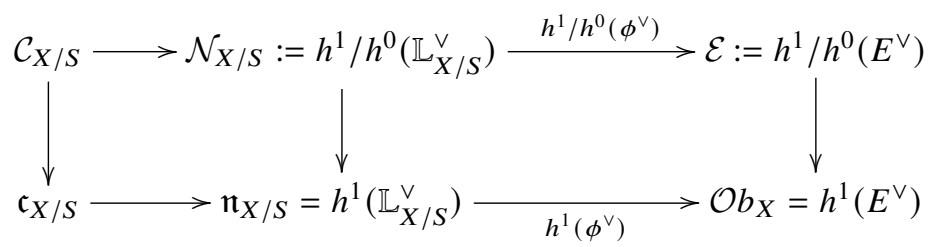

where $\mathcal{C}_{X / S}$ and $\mathcal{N}_{X / S}$ are the intrinsic normal cone and intrinsic normal sheaf of $X$ over $S$, respectively, while $\mathfrak{c}_{X / S}$ and $\mathfrak{n}_{X / S}$ are their coarse moduli sheaves. All the horizontal arrows are closed embeddings.

Some interesting moduli spaces in algebraic geometry, such as the moduli space of derived category objects, do not admit a perfect obstruction theory. In [16], we introduced a weaker notion with which moduli stacks for generalized Donaldson-Thomas invariants are equipped.

Definition 2.10. (Almost perfect obstruction theory) Let $X \rightarrow S$ be a morphism, where $X$ is a DeligneMumford stack of finite presentation and $S$ is a smooth Artin stack of pure dimension. An almost perfect obstruction theory $\phi$ consists of an étale covering $\left\{X_{\alpha} \rightarrow X\right\}_{\alpha \in A}$ of $X$ and perfect obstruction theories $\phi_{\alpha}: E_{\alpha} \rightarrow \mathbb{L}_{X_{\alpha} / S}$ of $X_{\alpha}$ over $S$ such that the following hold: 
1. For each pair of indices $\alpha, \beta$, there exists an isomorphism

$$
\psi_{\alpha \beta}:\left.\left.\mathcal{O} b_{X_{\alpha}}\right|_{\alpha \beta} \longrightarrow \mathcal{O} b_{X_{\beta}}\right|_{X_{\alpha \beta}}
$$

so that the collection $\left\{\mathcal{O} b_{X_{\alpha}}=h^{1}\left(E_{\alpha}^{\vee}\right), \psi_{\alpha \beta}\right\}$ gives descent data of a sheaf $\mathcal{O} b_{X}$, called the obstruction sheaf, on $X$.

2. For each pair of indices $\alpha, \beta$, there exists an étale covering $\left\{V_{\lambda} \rightarrow X_{\alpha \beta}\right\}_{\lambda \in \Gamma}$ of $X_{\alpha \beta}=X_{\alpha} \times_{X} X_{\beta}$ such that for any $\lambda$, the perfect obstruction theories $\left.E_{\alpha}\right|_{V_{\lambda}}$ and $\left.E_{\beta}\right|_{V_{\lambda}}$ are isomorphic and compatible with $\psi_{\alpha \beta}$. This means there exists an isomorphism

$$
\eta_{\alpha \beta \lambda}:\left.\left.E_{\alpha}\right|_{V_{\lambda}} \longrightarrow E_{\beta}\right|_{V_{\lambda}}
$$

in $D\left(V_{\lambda}\right)$ fitting in a commutative diagram

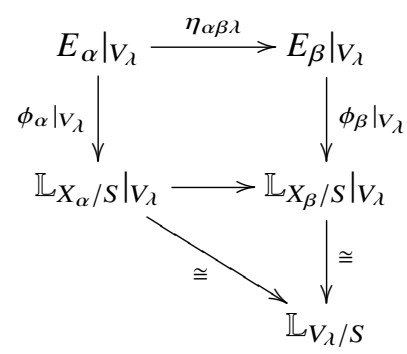

which moreover satisfies $h^{1}\left(\eta_{\alpha \beta \lambda}^{\vee}\right)=\left.\psi_{\alpha \beta}^{-1}\right|_{V_{\lambda}}$.

Suppose that the morphism $X \rightarrow S$ admits an almost perfect obstruction theory. Then the definition implies that the closed embeddings given in diagram (2.4)

$$
h^{1}\left(\phi_{\alpha}^{\vee}\right): \mathfrak{n}_{U_{\alpha} / S} \longrightarrow \mathcal{O} b_{X_{\alpha}}
$$

glue to a global closed embedding

$$
j_{\phi}: \mathfrak{n}_{X / S} \longrightarrow \mathcal{O} b_{X}
$$

of sheaf stacks over $X$. Therefore, the coarse intrinsic normal cone stack $\mathfrak{c}_{X / S}$ embeds as a closed substack into the sheaf stack $\mathcal{O} b_{X}$.

Definition 2.11. [16] (Virtual structure sheaf) Let $X \rightarrow S$ be as above, together with an almost perfect obstruction theory $\phi: X \rightarrow S$. The virtual structure sheaf of $X$ associated with $\phi$ is defined as

$$
\left[\mathcal{O}_{X}^{\mathrm{vir}}\right]:=0_{\mathcal{O} b_{X}}^{!}\left[\mathcal{O}_{\mathfrak{c}_{X / S}}\right] \in K_{0}(X)
$$

It is straightforward to generalize Definition 2.10 to the relative setting of a morphism $X \rightarrow Y$ of Deligne-Mumford stacks, by considering an étale cover $\left\{Y_{\alpha} \rightarrow Y\right\}$ and relative perfect obstruction theories on the morphisms $X_{\alpha}=X \times_{Y} Y_{\alpha} \rightarrow Y_{\alpha}$. We leave the detail to the reader.

In the subsequent sections, we will generalize the torus localization theorem [7, 26], the cosection localization theorem $[12,14]$ and the wall-crossing formula [5] to the virtual structure sheaves associated with almost perfect obstruction theories.

\section{Functorial behavior of coherent sheaves on sheaf stacks}

In this section, we investigate the functorial behavior of coherent sheaves on the sheaf stack $\mathcal{F}$. These functoriality properties will be our fundamental tools in generalizing the localization theorems to almost perfect obstruction theories in the subsequent sections. 
We will first establish a descent theory for coherent sheaves on $\mathcal{F}$. Using this, we will prove that there are pullbacks of a coherent sheaf $\mathcal{A}$ on $\mathcal{F}$ by a morphism $\tau: Y \rightarrow X$ of the base and by a surjective homomorphism $f: \mathcal{G} \rightarrow \mathcal{F}$ of the sheaves as well as by an injective homomorphism $f: \mathcal{G} \rightarrow \mathcal{F}$ with locally free quotient $L=\mathcal{F} / \mathcal{G}$.

Moreover, we will see that there are (higher) pushforwards of a coherent sheaf $\mathcal{A}$ on $\tau^{*} \mathcal{F}$ to coherent sheaves $R^{i} \tau_{*} \mathcal{A}$ on $\mathcal{F}$ for any proper morphism $\tau: Y \rightarrow X$. When $\mathcal{G} \rightarrow \mathcal{F}$ is an injective homomorphism of coherent sheaves on $X$ with locally free quotient, we will define the pushforward of a coherent sheaf on $\mathcal{G}$ to $\mathcal{F}$. These pushforwards and pullbacks satisfy the expected adjunction properties.

\subsection{Coherent descent theory}

Let $f: \mathcal{G} \rightarrow \mathcal{F}$ be a surjective homomorphism of coherent sheaves on a Deligne-Mumford stack $X$. Then any local chart

$$
Q=\left(\rho: U \rightarrow X, r_{E}: E \rightarrow \mathcal{G}\right)
$$

of $\mathcal{G}$ induces the local chart

$$
f_{*} Q=\left(\rho: U \rightarrow X, f_{*} r_{E}: E \rightarrow \mathcal{G} \rightarrow \mathcal{F}\right)
$$

of $\mathcal{F}$. Thus a coherent sheaf $\mathcal{A} \in \operatorname{Coh}(\mathcal{F})$ gives a coherent sheaf $\mathcal{A}_{f_{*} Q} \in \operatorname{Coh}(E)$ for every local chart $Q$ of $\mathcal{G}$. It is easy to see that this assignment is a coherent sheaf on $\mathcal{G}$, denoted by $f^{*} \mathcal{A}$ or $\left.\mathcal{A}\right|_{\mathcal{G}}$. In this way, we obtain a functor

$$
f^{*}: \operatorname{Coh}(\mathcal{F}) \longrightarrow \operatorname{Coh}(\mathcal{G})
$$

and a homomorphism

$$
f^{*}: K_{0}(\mathcal{F}) \longrightarrow K_{0}(\mathcal{G})
$$

since $f^{*}$ preserves exact sequences. From the construction, it is straightforward that if $g: \mathcal{G}^{\prime} \rightarrow \mathcal{G}$ is another surjective homomorphism, then we have the equality

$$
(f \circ g)^{*}=g^{*} \circ f^{*} .
$$

Using the above pullback functor, we can develop a descent formalism for sheaves on sheaf stacks. To begin with, it is convenient to extend the notion of local charts.

Definition 3.1. (Coherent chart) Let $\mathcal{F}$ be a coherent sheaf on a Deligne-Mumford stack $X$. A coherent chart on the sheaf stack $\mathcal{F}$ is the datum of a quadruple

$$
\mathcal{P}=\left(U, \rho, \mathcal{G}, r_{\mathcal{G}}\right)
$$

where $U$ is a scheme, $\rho: U \rightarrow X$ is an étale morphism and $r_{\mathcal{G}}: \mathcal{G} \rightarrow \rho^{*} \mathcal{F}$ is a surjective homomorphism of coherent sheaves on $U$.

Of course, when $\mathcal{G}$ is locally free, a coherent chart is the same as a local chart.

Morphisms between coherent charts are defined in the same way as morphisms between local charts. The main advantage of coherent charts is that one has a natural fiber product.

Definition 3.2. (Fiber product of coherent charts) Let $\mathcal{P}=\left(U, \rho, \mathcal{G}, r_{\mathcal{G}}\right)$ and $\mathcal{P}^{\prime}=\left(U^{\prime}, \rho^{\prime}, \mathcal{G}^{\prime}, r_{\mathcal{G}^{\prime}}\right)$ be two coherent charts for $\mathcal{F}$. Let $V=U \times_{X} U^{\prime}$ and $\rho_{V}: V \rightarrow X$ the natural map to $X$. Using (2.1), we have the fiber product

$$
\mathcal{G} \times_{\mathcal{F}} \mathcal{G}^{\prime}:=\left.\mathcal{G}\right|_{V} \times\left._{\left.\mathcal{F}\right|_{V}} \mathcal{G}^{\prime}\right|_{V}
$$


Denote the induced surjection $\mathcal{G} \times\left.{ }_{\mathcal{F}} \mathcal{G}^{\prime} \rightarrow \mathcal{F}\right|_{V}$ by $r_{\mathcal{G}} \times{ }_{\mathcal{F}} r_{\mathcal{G}^{\prime}}$. The coherent chart $\left(V, \rho_{V}, \mathcal{G} \times{ }_{\mathcal{F}} G^{\prime}, r_{\mathcal{G}} \times{ }_{\mathcal{F}} r_{\mathcal{G}^{\prime}}\right)$ is called the fiber product of the coherent charts $\mathcal{P}$ and $\mathcal{P}^{\prime}$ and denoted by $\mathcal{P} \times_{\mathcal{F}} \mathcal{P}^{\prime}$. There are natural morphisms of coherent charts $\mathcal{P} \times_{\mathcal{F}} \mathcal{P}^{\prime} \rightarrow \mathcal{P}$ and $\mathcal{P} \times_{\mathcal{F}} \mathcal{P}^{\prime} \rightarrow \mathcal{P}^{\prime}$.

Remark 3.3. If $\mathcal{P}$ and $\mathcal{P}^{\prime}$ are local charts, then it is easy to see that a common roof of $\mathcal{P}$ and $\mathcal{P}^{\prime}$ by Definition 2.4 is a local chart for the fiber product $\mathcal{P} \times{ }_{\mathcal{F}} \mathcal{P}^{\prime}$.

Definition 3.4. (Atlas on $\mathcal{F}$ ) An atlas on a sheaf stack $\mathcal{F}$ is a collection

$$
\left\{\mathcal{P}_{\alpha}=\left(U_{\alpha}, \rho_{\alpha}, \mathcal{G}_{\alpha}, r_{\alpha}\right)\right\}_{\alpha \in \Gamma}
$$

of coherent charts on $\mathcal{F}$ such that the morphisms $\left\{\rho_{\alpha}: U_{\alpha} \rightarrow X\right\}$ give an étale cover of $X$. We write $U_{\alpha \beta}=U_{\alpha} \times_{X} U_{\beta}$ and $\mathcal{P}_{\alpha \beta}=\left(U_{\alpha \beta}, \rho_{\alpha \beta}, \mathcal{G}_{\alpha \beta}, r_{\alpha \beta}\right)$ for the fiber product $\mathcal{P}_{\alpha} \times_{\mathcal{F}} \mathcal{P}_{\beta}$. We use similar notation $U_{\alpha \beta \gamma}$ and $\mathcal{P}_{\alpha \beta \gamma}$ for triples of indices.

If $\mathcal{A}$ is a coherent sheaf on $\mathcal{F}$ and $\left\{\mathcal{P}_{\alpha}\right\}$ is an atlas on $\mathcal{F}$, then we have the pullbacks

$$
\mathcal{A}_{\alpha} \in \operatorname{Coh}\left(\mathcal{G}_{\alpha}\right)
$$

of $\mathcal{A}$ to $\mathcal{G}_{\alpha}$ by the surjections $r_{\alpha}:\left.\mathcal{G}_{\alpha} \rightarrow \mathcal{F}\right|_{U_{\alpha}}$. Moreover, the pullbacks $\mathcal{A}_{\alpha}$ and $\mathcal{A}_{\beta}$ to $\mathcal{G}_{\alpha \beta}$ are isomorphic to $\left.\mathcal{A}\right|_{\mathcal{G}_{\alpha \beta}}$. By (3.3), we have the isomorphisms

$$
g_{\alpha \beta}:\left.\left.\left.\mathcal{A}_{\alpha}\right|_{\mathcal{G}_{\alpha \beta}} \stackrel{=}{\longrightarrow} \mathcal{A}\right|_{\mathcal{G}_{\alpha \beta}} \stackrel{=}{=} \mathcal{A}_{\beta}\right|_{\mathcal{G}_{\alpha \beta}},
$$

which satisfy the equality

$$
\left.\left.\left.g_{\gamma \alpha}\right|_{\mathcal{G}_{\alpha \beta \gamma}} \circ g_{\beta \gamma}\right|_{\mathcal{G}_{\alpha \beta \gamma}} \circ g_{\alpha \beta}\right|_{\mathcal{G}_{\alpha \beta \gamma}}=\mathrm{id} .
$$

Definition 3.5. (Descent datum) A descent datum on a sheaf stack $\mathcal{F}$ consists of

(a) An atlas $\left\{\mathcal{P}_{\alpha}=\left(U_{\alpha}, \rho_{\alpha}, \mathcal{G}_{\alpha}, r_{\alpha}\right)\right\}_{\alpha \in A}$ on $\mathcal{F}$

(b) A coherent sheaf $\mathcal{A}_{\alpha}$ on the sheaf stack $\mathcal{G}_{\alpha}$ for every index $\alpha$

(c) An isomorphism

$$
g_{\alpha \beta}:\left.\left.\mathcal{A}_{\alpha}\right|_{\mathcal{G}_{\alpha \beta}} \longrightarrow \mathcal{A}_{\beta}\right|_{\mathcal{G}_{\alpha \beta}}
$$

of coherent sheaves on the sheaf stack $\mathcal{G}_{\alpha \beta}$ for every pair $\alpha, \beta$,

such that the cocycle condition (3.5) holds for every triple $\alpha, \beta, \gamma$ of indices

We say that a descent datum is effective if there exists a coherent sheaf $\mathcal{A}$ on $\mathcal{F}$ such that we have $\mathcal{A}_{\alpha}=\left.\mathcal{A}\right|_{\mathcal{G}_{\alpha}}$ for all $\alpha$ and the $g_{\alpha \beta}$ are the naturally induced isomorphisms (3.4) on fiber products. We then say that the datum descends or glues to the sheaf $\mathcal{A}$.

For example, let $f: \mathcal{G} \rightarrow \mathcal{F}$ be a surjective homomorphism of coherent sheaves on a DeligneMumford stack $X$. The single chart (id :X $\rightarrow X, f: \mathcal{G} \rightarrow \mathcal{F}$ ) is then an atlas for $\mathcal{F}$. The fiber product $\mathcal{G} \times_{\mathcal{F}} \mathcal{G}$ fits into the commutative diagram

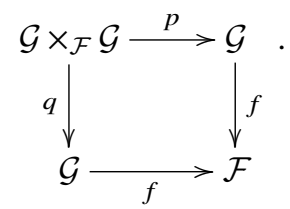

A descent datum for this atlas consists of a coherent sheaf $\mathcal{B}$ on $\mathcal{G}$ and an isomorphism $g: p^{*} \mathcal{B} \rightarrow q^{*} \mathcal{B}$. For a local chart $\left(\rho: U \rightarrow X, r_{E}: E \rightarrow \rho^{*} \mathcal{F}\right)$ with affine base $U$, we pick a surjection $W \rightarrow E \times\left._{\left.\mathcal{F}\right|_{U}} \mathcal{G}\right|_{U}$ from a locally free sheaf $W$ on $U$ so that we have surjections

$$
r_{W}: W \longrightarrow E \times\left.\left._{\left.\mathcal{F}\right|_{U}} \mathcal{G}\right|_{U} \longrightarrow \mathcal{G}\right|_{U}, \quad W \times_{E} W \longrightarrow \mathcal{G} \times\left._{\mathcal{F}} \mathcal{G}\right|_{U}
$$


and a fiber diagram

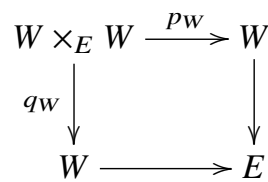

lying over (3.6). As $\mathcal{B} \in \operatorname{Coh}(\mathcal{G})$, we have a coherent sheaf $\mathcal{B}_{W}$ on $W$ and an isomorphism $p_{W}^{*} \mathcal{B}_{W} \cong$ $q_{W}^{*} \mathcal{B}_{W}$. Since $W \rightarrow E$ is a surjective homomorphism of vector bundles, the coherent sheaf $\mathcal{B}_{W}$ descends to a coherent sheaf $\mathcal{B}_{E}$ on $E$. It is easy to see that $\mathcal{B}_{E}$ is independent of the choice of $W$ and we have a coherent sheaf $\mathcal{B}_{E}$ for any local chart of $\mathcal{F}$ by considering an affine cover of the base scheme $U$. In this way, we obtain a coherent sheaf on $\mathcal{F}$.

By the same argument, we deduce the following.

Proposition 3.6. (Descent for sheaves on $\mathcal{F}$ ) Any descent datum on a sheaf stack $\mathcal{F}$ is effective and descends to a coherent sheaf on $\mathcal{F}$, which is uniquely determined (up to canonical isomorphism).

Proof. This is a standard descent argument using smooth descent for sheaves on schemes repeating the reasoning we saw above on an atlas for $\mathcal{F}$ and keeping track of all the indices involved. We leave the details to the reader.

\subsection{Pullbacks of coherent sheaves on sheaf stacks}

In this subsection, we will see more ways to pull back coherent sheaves or $K$-theory classes, other than (3.1) and (3.2).

Let $\mathcal{F}$ be a coherent sheaf on a Deligne-Mumford stack $X$. Then all local charts of the sheaf stack $\mathcal{F}$ form an atlas in the obvious way. Suppose we have a short exact sequence

$$
0 \longrightarrow \mathcal{G} \stackrel{f}{\longrightarrow} \mathcal{F} \longrightarrow L \longrightarrow 0
$$

of coherent sheaves on $X$ with $L$ locally free. Then any local chart $Q=\left(U, \rho, E, r_{E}\right)$ of $\mathcal{F}$ gives rise to the commutative diagram of exact sequences

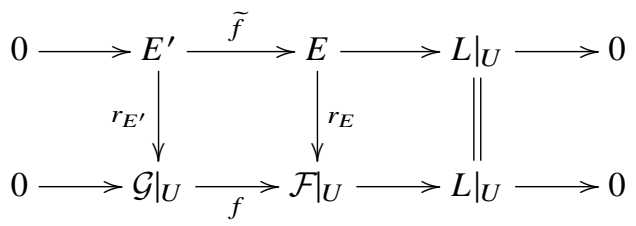

whose vertical arrows are all surjective. Note that $E^{\prime}$ is locally free, and it is straightforward to see that the local charts $Q^{\prime}=\left(U, \rho, E^{\prime}, r_{E^{\prime}}\right)$ form an atlas on the sheaf stack $\mathcal{G}$.

Given a coherent sheaf $\mathcal{A}_{Q}$ on $E$, we can pull it back to $E^{\prime}$ to get a coherent sheaf $\mathcal{A}_{Q^{\prime}}^{\prime}=\widetilde{f}^{*} \mathcal{A}_{Q}$. If $\mathcal{A} \in \operatorname{Coh}(\mathcal{F})$, we then obtain a descent datum $\left\{\mathcal{A}_{Q^{\prime}}^{\prime} \in \operatorname{Coh}\left(E^{\prime}\right)\right\}$ with the atlas $\left\{Q^{\prime}\right\}$ on $\mathcal{G}$. By Proposition 3.6, we thus obtain a sheaf $\mathcal{A}^{\prime}$ on $\mathcal{G}$, which we denote by $f^{*} \mathcal{A} \in \operatorname{Coh}(\mathcal{G})$. Likewise, we can pull back homomorphisms in $\operatorname{Coh}(\mathcal{F})$ to $\operatorname{Coh}(\mathcal{G})$. We thus obtain the pullback functor

$$
f^{*}: \operatorname{Coh}(\mathcal{F}) \longrightarrow \operatorname{Coh}(\mathcal{G})
$$

for an injective homomorphism $f: \mathcal{G} \rightarrow \mathcal{F}$ of coherent sheaves on $X$ whose cokernel $L=\mathcal{F} / \mathcal{G}$ is locally free.

Unfortunately, this functor $f^{*}$ is not exact and hence does not induce a homomorphism on $K$-groups. To get an exact functor, we should use the left derived pullback: letting $\pi_{E}: E \rightarrow U$ denote the bundle projection, we have a tautological section $s$ of $\pi_{E}^{*} L$ whose vanishing locus is $E^{\prime}$. 
We then have the Koszul complex $\mathcal{K}(L)$, and the homology sheaves of the complex $\mathcal{K}(L) \otimes \mathcal{A}_{Q}$ for each local chart $Q$ of $\mathcal{F}$ give us descent data with respect to the atlas $\left\{Q^{\prime}\right\}$ on $\mathcal{G}$. By Proposition 3.6, we get coherent sheaves

$$
L_{0} f^{*} \mathcal{A}=f^{*} \mathcal{A}, \quad L_{1} f^{*} \mathcal{A}, \quad L_{2} f^{*} \mathcal{A}, \quad \cdots
$$

on $\mathcal{G}$ (with degrees up to the rank of $L$ ), where each $L_{i} f^{*} \mathcal{A}$ is the descent of the homology sheaves in degree $-i$ of the complexes $\mathcal{K}(L) \otimes \mathcal{A}_{Q}$ for local charts $Q$. We thus obtain the pullback homomorphism

$$
f^{*}: K_{0}(\mathcal{F}) \longrightarrow K_{0}(\mathcal{G}), \quad[\mathcal{A}] \mapsto \sum_{i \geq 0}(-1)^{i}\left[L_{i} f^{*} \mathcal{A}\right]
$$

It is straightforward to check that (3.10) is well defined.

The pullback (3.10) is compatible with the Gysin map $0_{\mathcal{F}}^{!}$as follows.

Lemma 3.7. Let $f: \mathcal{G} \rightarrow \mathcal{F}$ be an injective homomorphism of coherent sheaves on $X$ whose cokernel $L=\mathcal{F} / \mathcal{G}$ is locally free. Then we have the identity

$$
0_{\mathcal{G}}^{!} \circ f^{*}=0_{\mathcal{F}}^{!}
$$

Proof. For any affine local chart $Q=\left(U, \rho, E, r_{E}\right)$, we can split $E$ as the direct sum $E^{\prime} \oplus L$, and we have an induced isomorphism $\mathcal{K}(E) \cong \mathcal{K}\left(E^{\prime}\right) \otimes \mathcal{K}(L)$ of Koszul complexes. Given a coherent sheaf $\mathcal{A}$ on $\mathcal{F}$, we then obtain an isomorphism

$$
\mathcal{K}(E) \otimes \mathcal{A}_{Q} \cong \mathcal{K}\left(E^{\prime}\right) \otimes\left(\mathcal{K}(L) \otimes \mathcal{A}_{Q}\right)
$$

The spectral sequence for the double complex on the right-hand side gives an equality in $K$-theory for any $\ell \geq 0$

$$
\left[\mathcal{H}_{Q}^{\ell}(\mathcal{A})\right]=\sum_{i+j=\ell}(-1)^{j}\left[\mathcal{H}_{Q^{\prime}}^{i}\left(L_{j} f^{*} \mathcal{A}\right)\right] \in K_{0}(U) .
$$

Since the spectral sequence maps are functorial with respect to morphisms between local charts and independent of the choice of splitting of $E$, the above equality is true globally for Koszul homology sheaves using descent, and the lemma follows.

We can also pull back coherent sheaves on a sheaf stack by a morphism of the underlying DeligneMumford stack. Let $\tau: Y \rightarrow X$ be a morphism of Deligne-Mumford stacks and $\mathcal{F}$ be a coherent sheaf on $X$. As we saw above, local charts $Q=\left(U, \rho, E, r_{E}\right)$ of the sheaf stack $\mathcal{F}$ form an atlas on $\mathcal{F}$. The pullbacks

$$
Q^{\prime}=\left(\rho^{\prime}: U^{\prime}=Y \times_{X} U \rightarrow Y, r_{E^{\prime}}=\tau^{*} r_{E}: E^{\prime}=\tau^{*} E \rightarrow \tau^{*} \mathcal{F}\right)
$$

form an atlas of the sheaf stack $\tau^{*} \mathcal{F}$ on $Y$. By the induced morphism $\widetilde{\tau}: E^{\prime}=\tau^{*} E \rightarrow E$, we can pull back coherent sheaves on $E$ to $E^{\prime}$. If $\mathcal{A} \in \operatorname{Coh}(\mathcal{F})$ is a coherent sheaf on $\mathcal{F}$, the coherent sheaves $\left\{\widetilde{\tau}^{*} \mathcal{A}_{Q} \in \operatorname{Coh}\left(E^{\prime}\right)\right\}$ form a descent datum and hence glue to a coherent sheaf $\tau^{*} \mathcal{A}$ on $\tau^{*} \mathcal{F}$. The same holds for homomorphisms in $\operatorname{Coh}(\mathcal{F})$. We thus obtain the pullback functor

$$
\tau^{*}: \operatorname{Coh}(\mathcal{F}) \longrightarrow \operatorname{Coh}\left(\tau^{*} \mathcal{F}\right)
$$

for a morphism $\tau: Y \rightarrow X$ of Deligne-Mumford stacks and a sheaf stack $\mathcal{F}$ over $X$.

If $\tau$ is flat, then $\widetilde{\tau}: E^{\prime} \rightarrow E$ is flat and $\widetilde{\tau}^{*}$ is exact. Hence we obtain a homomorphism

$$
\tau^{*}: K_{0}(\mathcal{F}) \rightarrow K_{0}\left(\tau^{*} \mathcal{F}\right)
$$

when $\tau: Y \rightarrow X$ is flat.

The pullback $\tau^{*}$ by a flat morphism is compatible with the Gysin maps. 
Lemma 3.8. Let $\tau: Y \rightarrow X$ be a flat morphism of Deligne-Mumford stacks and $\mathcal{F}$ be a coherent sheaf on $X$. Then the pullback (3.13) fits into the commutative diagram

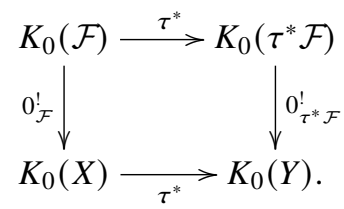

Proof. The lemma follows from the isomorphism $\tau^{*} \mathcal{K}(E) \cong \mathcal{K}\left(\tau^{*} E\right)$ of Koszul complexes.

\subsection{Pushforwards of coherent sheaves on sheaf stacks}

In this subsection, we will consider pushforwards on sheaf stacks.

Suppose we have a short exact sequence

$$
0 \longrightarrow \mathcal{G} \stackrel{f}{\longrightarrow} \mathcal{F} \longrightarrow L \longrightarrow 0
$$

of coherent sheaves on a Deligne-Mumford stack $X$ with $L$ locally free. For any local chart $Q=$ $\left(U, \rho, E, r_{E}\right)$, we have a commutative diagram (3.8) of exact sequences, and $Q^{\prime}=\left(U, \rho, E^{\prime}, r_{E^{\prime}}\right)$ is a local chart for $\mathcal{G}$. Given a coherent sheaf $\mathcal{B} \in \operatorname{Coh}(\mathcal{G})$, we have a coherent sheaf $\mathcal{B}_{Q^{\prime}}$ on $E^{\prime}$ and its pushforward $\widetilde{f}_{*} \mathcal{B}_{Q^{\prime}} \in \operatorname{Coh}(E)$ by the closed immersion $\widetilde{f}$. By the base change property, the assignment $Q \mapsto \widetilde{f}_{*} \mathcal{B}_{Q^{\prime}}$ is a coherent sheaf on $\mathcal{F}$, which we denote by $f_{*} \mathcal{B} \in \operatorname{Coh}(\mathcal{F})$. Likewise, we can push forward a homomorphism in $\operatorname{Coh}(\mathcal{G})$ to $\operatorname{Coh}(\mathcal{F})$ by $f$. We thus obtain a functor

$$
f_{*}: \operatorname{Coh}(\mathcal{G}) \longrightarrow \operatorname{Coh}(\mathcal{F})
$$

which is exact as $\widetilde{f}$ is a closed immersion. So we have a homomorphism

$$
f_{*}: K_{0}(\mathcal{G}) \longrightarrow K_{0}(\mathcal{F})
$$

for an injective homomorphism $f: \mathcal{G} \rightarrow \mathcal{F}$ of coherent sheaves on $X$ with locally free cokernel $L=\mathcal{F} / \mathcal{G}$.

By construction, the natural adjunction homomorphisms

$$
\mathcal{A}_{Q} \longrightarrow \widetilde{f_{*}} \widetilde{f}^{*} \mathcal{A}_{Q}, \quad \widetilde{f^{*}} \widetilde{f}_{*} \mathcal{B}_{Q^{\prime}} \longrightarrow \mathcal{B}_{Q^{\prime}}
$$

induce the natural homomorphisms

$$
\mathcal{A} \longrightarrow f_{*} f^{*} \mathcal{A}, \quad f^{*} f_{*} \mathcal{B} \longrightarrow \mathcal{B}
$$

in $\operatorname{Coh}(\mathcal{F})$ and $\operatorname{Coh}(\mathcal{G})$, respectively.

Let $\tau: Y \rightarrow X$ be a proper morphism of Deligne-Mumford stacks. Let $\mathcal{F}$ be a coherent sheaf on $X$. A local chart $Q=\left(U, \rho, E, r_{E}\right)$ of $\mathcal{F}$ induces a local chart $Q^{\prime}$, defined by (3.11), of the sheaf stack $\tau^{*} \mathcal{F}$ and a proper morphism

$$
\widetilde{\tau}: E^{\prime}=\tau^{*} E \rightarrow E .
$$

Let $\mathcal{B} \in \operatorname{Coh}\left(\tau^{*} \mathcal{F}\right)$ be a coherent sheaf on $\tau^{*} \mathcal{F}$. Then we have the coherent sheaves

$$
\widetilde{\tau}_{*} \mathcal{B}_{Q^{\prime}}, \quad R^{i} \widetilde{\tau}_{*} \mathcal{B}_{Q^{\prime}} \in \operatorname{Coh}(E)
$$

by taking the direct and higher direct images. By the base chage property [8, III, Proposition 9.3], the assignments

$$
Q \mapsto \widetilde{\tau}_{*} \mathcal{B}_{Q^{\prime}}, \quad Q \mapsto R^{i} \widetilde{\tau}_{*} \mathcal{B}_{Q^{\prime}}
$$


are coherent sheaves on $\mathcal{F}$, which we denote by $\tau_{*} \mathcal{B}=R^{0} \tau_{*} \mathcal{B}$ and $R^{i} \tau_{*} \mathcal{B}$, respectively. We thus obtain functors

$$
\tau_{*}=R^{0} \tau_{*}, R^{i} \tau_{*}: \operatorname{Coh}\left(\tau^{*} \mathcal{F}\right) \longrightarrow \operatorname{Coh}(\mathcal{F})
$$

for $i>0$, which give us the homomorphism

$$
\tau_{*}: K_{0}\left(\tau^{*} \mathcal{F}\right) \longrightarrow K_{0}(\mathcal{F}), \quad[\mathcal{B}] \mapsto \sum_{i}(-1)^{i}\left[R^{i} \tau_{*} \mathcal{B}\right]
$$

for a proper morphism $\tau: Y \rightarrow X$.

Lemma 3.9. For a proper morphism, the diagram

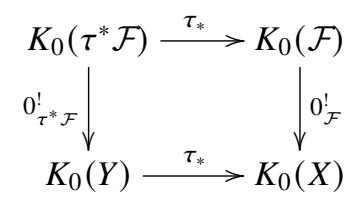

commutes.

Proof. The lemma follows from Definition 2.9 and the projection formula [8, III, Exer. 8.3].

As above, the usual adjunction homomorphisms give us homomorphisms

$$
\mathcal{A} \longrightarrow \tau_{*} \tau^{*} \mathcal{A}, \quad \tau^{*} \tau_{*} \mathcal{B} \rightarrow \mathcal{B}
$$

in $\operatorname{Coh}(\mathcal{F})$ and $\operatorname{Coh}\left(\tau^{*} \mathcal{F}\right)$, respectively.

\section{Cosection localization}

Let $X \rightarrow S$ be a morphism, where $X$ is a Deligne-Mumford stack of finite type and $S$ a smooth quasiprojective curve, together with a perfect obstruction theory $\phi$ and a cosection $\sigma: \mathcal{O} b_{\phi} \rightarrow \mathcal{O}_{X}$. In [12] and [14], it is shown that the virtual fundamental cycle $[X]^{\text {vir }} \in A_{*}(X)$ and virtual structure sheaf $\left[\mathcal{O}_{X}^{\mathrm{vir}}\right] \in K_{0}(X)$ localize to the vanishing locus $X(\sigma)$ of $\sigma$, being the pushforward of localized classes in $A_{*}(X(\sigma))$ and $K_{0}(X(\sigma))$, respectively, in a canonical way.

In this section, we prove the analogous cosection localization for virtual structure sheaves induced by an almost perfect obstruction theory.

\subsection{Cosection localization for perfect obstruction theory}

Let $X \rightarrow S$ be as above. Let $\rho: U \rightarrow X$ be an étale morphism from a scheme $U$, and let $\phi: E \rightarrow \mathbb{L}_{U / S}^{\geq-1}$ be a perfect obstruction theory with $E=\left[E^{-1} \rightarrow E^{0}\right]$ a global resolution by vector bundles. Moreover, suppose that we have a morphism

$$
\sigma: \mathcal{O} b_{U} \longrightarrow \mathrm{O}_{U}
$$

which, following [12], we refer to as a cosection.

Let $\sigma$ be the composition $E_{1}=\left(E^{-1}\right)^{\vee} \rightarrow \mathcal{O} b_{U} \rightarrow \mathcal{G}_{U}, U(\sigma)$ the vanishing locus of $\sigma, U^{\circ}=$ $U-U(\bar{\sigma})$ and

$$
E_{1}(\sigma)=\left.E_{1}\right|_{U(\sigma)} \cup \operatorname{ker}\left(\left.\underline{\sigma}\right|_{U^{\circ}}:\left.E_{1}\right|_{U^{\circ}} \longrightarrow \sigma_{U^{\circ}}\right)
$$


In [14], the authors define a localized Gysin map

$$
0_{E_{1}, \sigma}^{!}: K_{0}\left(E_{1}(\sigma)\right) \rightarrow K_{0}(U(\sigma))
$$

We recall the construction. Let $\tau: \widetilde{U} \rightarrow U$ be the blowup of $U$ along $U(\sigma)$ with exceptional divisor $D$. If $\widetilde{E}_{1}=\tau^{*} E_{1}$, we have an induced surjection

$$
\widetilde{\widetilde{\sigma}}: \widetilde{E}_{1} \longrightarrow \sigma_{\widetilde{U}}(-D) .
$$

Let $E_{1}^{\prime}=\operatorname{ker} \underline{\widetilde{\sigma}}$ and

$$
\widetilde{\tau}: E_{1}^{\prime} \longrightarrow E_{1}(\sigma)
$$

be the morphism induced from $\widetilde{E}_{1} \rightarrow E_{1}$.

For any coherent sheaf $\mathcal{A}$ on $E_{1}(\sigma)$, we have a natural morphism by adjunction

$$
\eta_{\mathcal{A}}: \mathcal{A} \longrightarrow \widetilde{\tau}_{*} \widetilde{\tau}^{*} \mathcal{A}
$$

Since $\eta_{\mathcal{A}}$ is an isomorphism over $U^{\circ}$, the sheaves $\operatorname{ker}\left(\eta_{\mathcal{A}}\right), \operatorname{coker}\left(\eta_{\mathcal{A}}\right)$ and $R^{i} \widetilde{\tau}_{*} \widetilde{\tau}^{*} \mathcal{A}$ for $i \geq 1$ are supported on $\left.E_{1}\right|_{U(\sigma)}$.

We may therefore define

$$
R_{\mathcal{A}}:=\left[\operatorname{ker}\left(\eta_{\mathcal{A}}\right)\right]-\left[\operatorname{coker}\left(\eta_{\mathcal{A}}\right)\right]-\sum_{i \geq 1}(-1)^{i}\left[R^{i} \widetilde{\tau}_{*} \widetilde{\tau}^{*} \mathcal{A}\right] \in K_{0}\left(\left.E_{1}\right|_{U(\sigma)}\right) .
$$

Definition 4.1. [14] The cosection localized Gysin map is given by the formula

$$
0_{E_{1}, \sigma}^{!}[\mathcal{A}]:=\left(\left.\tau\right|_{D}\right)_{*}\left(D^{\vee} \cdot 0_{E_{1}^{\prime}}^{!}\left[\widetilde{\tau}^{*} \mathcal{A}\right]\right)+0_{\left.E_{1}\right|_{U(\sigma)}}^{!} R_{\mathcal{A}} \in K_{0}(U(\sigma)),
$$

where $D^{\vee} \cdot\left[\mathcal{A}^{\prime}\right]=\left[\sigma_{\widetilde{X}} \rightarrow \sigma_{\widetilde{X}}(D)\right] \otimes\left[\mathcal{A}^{\prime}\right]$.

Let $C_{1}:=\mathfrak{c}_{X / S} \times_{\mathcal{O} b_{X}} E_{1} \subset E_{1}$ be the obstruction cone of the perfect obstruction theory $\phi$. In [12], it is shown that $C_{1}$ has reduced support in $E_{1}(\sigma)$. Therefore, if we let $I$ denote the ideal sheaf of $\left(C_{1}\right)^{\text {red }} \subset C_{1}$, the sheaves $\mathcal{A}_{j}=I^{j} \sigma_{C_{1}} / I^{j+1} \sigma_{C_{1}}$ for $j \geq 0$ are naturally coherent sheaves on $E_{1}(\sigma)$, and moreover

$$
\left[\sigma_{C_{1}}\right]=\sum_{j \geq 0}\left[\mathcal{A}_{j}\right] \in K_{0}\left(E_{1}(\sigma)\right)
$$

where the summation is finite, since $I^{j}=0$ for large enough $j$.

Definition-Theorem 4.2. [14] The cosection localized virtual structure sheaf on $U$ is defined by

$$
\left[\mathcal{O}_{U, \text { loc }}^{\mathrm{vir}}\right]=0_{E_{1}, \sigma}^{!}\left[\mathcal{G}_{C_{1}}\right]:=\sum_{j \geq 0} 0_{E_{1}, \sigma}^{!}\left[\mathcal{A}_{j}\right] \in K_{0}(U(\sigma)) .
$$

It satisfies

$$
\iota_{*}\left[\mathcal{G}_{U, \mathrm{loc}}^{\mathrm{vir}}\right]=\left[\mathcal{G}_{U}^{\mathrm{vir}}\right] \in K_{0}(U),
$$

where $\iota: U(\sigma) \rightarrow U$ is the inclusion, is independent of the particular choice of global resolution for $E$ and deformation invariant.

The purpose of this section is to generalize Definition-Theorem 4.2 to the setting of almost perfect obstruction theories. 


\subsection{Intrinsic normal cone under a cosection}

Recall from Section 2.4 that under the assumptions of Definition 2.10, if $X \rightarrow S$ is equipped with an almost perfect obstruction theory $\phi$, we have the intrinsic normal sheaf $\mathfrak{c}_{X / S}$, which is a closed substack of the obstruction sheaf $O b_{X}$ of $\phi$. Let $\mathcal{F}=O b_{X}$ in the rest of this section.

For any local chart $Q=\left(U, \rho, E, r_{E}\right)$ of the sheaf stack $\mathcal{F}$, we have a Cartesian square

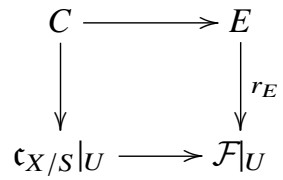

whose horizontal arrows are closed immersions. Hence $C$ is a closed subscheme of the vector bundle $E$, and its structure sheaf $\sigma_{C}$ is a coherent sheaf on $E$. It is obvious that the assignment $Q \mapsto \sigma_{C}$ is a coherent sheaf on the sheaf stack $\mathcal{F}$, which we denote by

$$
\sigma_{\mathfrak{c}_{X / S}} \in \operatorname{Coh}(\mathcal{F})
$$

Now suppose we have a cosection

$$
\sigma: \mathcal{F}=O b_{X} \longrightarrow \sigma_{X}
$$

of the obstruction sheaf. As mentioned above, it was proved in [12] that for any local chart $Q=(\rho: U \rightarrow$ $\left.X, r_{E}:\left.E \rightarrow \mathcal{F}\right|_{U}\right)$ of the sheaf stack $\mathcal{F}$, the cone $C=E \times\left._{\left.\mathcal{F}\right|_{U}} \mathfrak{c}_{X / S}\right|_{U} \subset E$ in (4.5) has reduced support in

$$
E(\sigma)=\left.E\right|_{U(\sigma)} \cup \operatorname{ker}\left(\left.E \stackrel{r_{E}}{\longrightarrow} \mathcal{F}\right|_{U} \stackrel{\sigma}{\longrightarrow} \bigcirc_{U}\right),
$$

where $U(\sigma)$ is the vanishing locus of $\sigma$ that is the closed subscheme of $U$ defined by the image of $\left.\sigma\right|_{U}:\left.\mathcal{F}\right|_{U}=\rho^{*} \mathcal{F} \rightarrow \mathcal{O}_{U}$. The closed substacks $E(\sigma)$ for local charts $Q=\left(U, \rho, E, r_{E}\right)$ define a closed substack that we denote by $\mathcal{F}(\sigma)$. We let $\underline{\operatorname{Coh}}(\mathcal{F})$ denote the set of isomorphism classes of coherent sheaves on the sheaf stack $\mathcal{F}$, and let

$$
\underline{\operatorname{Coh}}_{\sigma}(\mathcal{F})
$$

denote the subset of isomorphisms classes of coherent sheaves $\mathcal{A}$ on $\mathcal{F}$ with support in $\mathcal{F}(\sigma)$ : that is, for each local chart $Q=\left(U, \rho, E, r_{E}\right), \mathcal{A}_{Q}$ has support in $E(\sigma)$.

The assignment to a local chart $Q$ of the ideal sheaf $I_{Q}$ of $E(\sigma)$ on $E$ is a coherent sheaf $\mathcal{I}$, and there exist exact sequences

$$
0 \longrightarrow \mathcal{I}^{j+1} \mathcal{O}_{\mathfrak{c}_{X / S}} \longrightarrow \mathcal{I}^{j} \sigma_{\mathfrak{c}_{X / S}} \longrightarrow \mathcal{I}^{j} \circlearrowleft_{\mathfrak{c}_{X / S}} / \mathcal{I}^{j+1} \circlearrowleft_{\mathfrak{c}_{X / S}} \longrightarrow 0
$$

We let

$$
\mathcal{A}_{j}=\mathcal{I}^{j} \widehat{\sigma}_{\mathfrak{c}_{X / S}} / \mathcal{I}^{j+1} \widehat{O}_{\mathfrak{c}_{X / S}} \in \operatorname{Coh}(\mathcal{F})
$$

so that for any local chart $Q=\left(U, \rho, E, r_{E}\right)$ of $\mathcal{F},\left(\mathcal{A}_{j}\right)_{Q} \in \operatorname{Coh}(E)$ is a coherent sheaf supported in $E(\sigma)$. Note that the isomorphism class of $\mathcal{A}_{j}$ lies in $\underline{\mathrm{Coh}} \sigma(\mathcal{F})$ and

$$
\left[\widehat{G}_{\mathfrak{c}_{X / S}}\right]=\sum_{j}\left[\mathcal{A}_{j}\right] \in K_{0}(\mathcal{F})
$$

by the definition of the $\mathcal{A}_{j}$. 


\subsection{Cosection localized Gysin maps}

In this subsection, we will define a map

$$
0_{\mathcal{F}, \sigma}^{!}: \underline{\operatorname{Coh}}_{\sigma}(\mathcal{F}) \longrightarrow K_{0}(X(\sigma))
$$

where $X(\sigma)$ is the vanishing locus of the cosection $\sigma$. The cosection localized virtual structure sheaf $\left[\mathcal{G}_{X, \mathrm{loc}}^{\mathrm{vir}}\right] \in K_{0}(X(\sigma))$ will be defined by

$$
\left[\mathcal{G}_{X, \mathrm{loc}}^{\mathrm{vir}}\right]=\sum_{j} 0_{\mathcal{F}, \sigma}^{!}\left(\mathcal{A}_{j}\right)
$$

with $\mathcal{A}_{j}$ from (4.8). By construction, it will follow that the pushforward of $\left[\mathcal{O}_{X, \text { loc }}^{\mathrm{vir}}\right]$ by the inclusion $X(\sigma) \hookrightarrow X$ is the usual virtual structure sheaf $\left[\mathcal{O}_{X}^{\mathrm{vir}}\right] \in K_{0}(X)$.

Let $\tau: \widetilde{X} \rightarrow X$ be the blowup of $X$ along $X(\sigma)$. Let $D$ denote the exceptional divisor. The cosection $\sigma: \mathcal{F} \rightarrow \sigma_{X}$ lifts to a surjection $\tau^{*} \mathcal{F}=\left.\mathcal{F}\right|_{\tilde{X}} \rightarrow \sigma_{\widetilde{X}}(-D)$ whose kernel is denoted by $\mathcal{F}^{\prime}$, so that we have an exact sequence

$$
0 \longrightarrow \mathcal{F}^{\prime} \stackrel{f}{\longrightarrow} \tau^{*} \mathcal{F} \longrightarrow \sigma_{\widetilde{X}}(-D) \longrightarrow 0
$$

Let $\mathcal{A} \in \operatorname{Coh}(\mathcal{F})$ whose isomorphism class lies in $\underline{\operatorname{Coh}}_{\sigma}(\mathcal{F})$. By the pullback functors (3.9) and (3.12), we have

$$
\left[f^{*} \tau^{*} \mathcal{A}\right] \in K_{0}\left(\mathcal{F}^{\prime}\right) .
$$

By applying the Gysin map for $\mathcal{F}^{\prime}$ (Definition 2.9), we obtain

$$
0_{\mathcal{F}^{\prime}}^{!}\left[f^{*} \tau^{*} \mathcal{A}\right] \in K_{0}(\widetilde{X})
$$

Then we intersect it with $-D$ to obtain

$$
D^{\vee} \cdot 0_{\mathcal{F}}^{!}\left[f^{*} \tau^{*} \mathcal{A}\right]=\left[\circlearrowleft_{\widetilde{X}} \rightarrow \sigma_{\widetilde{X}}(D)\right] \otimes 0_{\mathcal{F}}\left[f^{*} \tau^{*} \mathcal{A}\right] \in K_{0}(D) .
$$

Now we push it down to $X(\sigma)$ by $\left.\tau\right|_{D}$ to obtain

$$
\left(\left.\tau\right|_{D}\right)_{*}\left(D^{\vee} \cdot 0_{\mathcal{F}^{\prime}}^{!}\left[f^{*} \tau^{*} \mathcal{A}\right]\right) \in K_{0}(X(\sigma)) .
$$

Let $\imath: X(\sigma) \rightarrow X$ and $\widetilde{\imath}: D \rightarrow \widetilde{X}$ denote the inclusions. Then

$$
\begin{gathered}
l_{*}\left(\left.\tau\right|_{D}\right)_{*}\left(D^{\vee} \cdot 0_{\mathcal{F}}^{!}\left[f^{*} \tau^{*} \mathcal{A}\right]\right)=\tau_{*} \widetilde{l}_{*}\left(D^{\vee} \cdot 0_{\mathcal{F}^{\mathcal{F}}}^{!}\left[f^{*} \tau^{*} \mathcal{A}\right]\right) \\
=\tau_{*} 0_{{ }_{\Theta_{\tilde{X}}(-D)}}^{!} 0_{\mathcal{F}^{\prime}}^{!}\left[f^{*} \tau^{*} \mathcal{A}\right]=\tau_{*} 0_{\tau^{*} \mathcal{F}}^{!}\left[f_{*} f^{*} \tau^{*} \mathcal{A}\right] \\
=0_{\mathcal{F}^{\prime}}^{!} \tau_{*}\left[f_{*} f^{*} \tau^{*} \mathcal{A}\right]=\sum_{i \geq 0}(-1)^{i} 0_{\mathcal{F}}^{!}\left[R^{i} \tau_{*} f_{*} f^{*} \tau^{*} \mathcal{A}\right] .
\end{gathered}
$$

Since $\mathcal{A}$ is a sheaf with support in $\mathcal{F}(\sigma)$ and $\tau$ is an isomorphism on $X-X(\sigma)$, we find that $\left.R^{i} \tau_{*} f_{*} f^{*} \tau^{*} \mathcal{A}\right|_{X-X(\sigma)}=0$ is a coherent sheaf on the closed substack $\left.\mathcal{F}\right|_{X(\sigma)}$ for $i>0$. Likewise, the kernel and cokernel of the natural homomorphism

$$
\eta_{\mathcal{A}}: \mathcal{A} \longrightarrow \tau_{*} f_{*} f^{*} \tau^{*} \mathcal{A}
$$


are coherent sheaves on $\left.\mathcal{F}\right|_{X(\sigma)}$. Let

$$
R_{\mathcal{A}}:=\left[\operatorname{ker}\left(\eta_{\mathcal{A}}\right)\right]-\left[\operatorname{coker}\left(\eta_{\mathcal{A}}\right)\right]-\sum_{i \geq 1}(-1)^{i}\left[R^{i} \tau_{*} f_{*} f^{*} \tau^{*} \mathcal{A}\right] \in K_{0}\left(\left.\mathcal{F}\right|_{X(\sigma)}\right)
$$

Then by Lemma 3.9, we have

$$
\begin{aligned}
& l_{*} 0_{\left.\mathcal{F}\right|_{X(\sigma)}}^{!} R_{\mathcal{A}}=0_{\mathcal{F}^{\prime}}^{!} l_{\mathcal{A}} \\
& =0_{\mathcal{F}}\left([\mathcal{A}]-\left[\tau_{*} f_{*} f^{*} \tau^{*} \mathcal{A}\right]-\sum_{i \geq 1}(-1)^{i}\left[R^{i} \tau_{*} f_{*} f^{*} \tau^{*} \mathcal{A}\right]\right) \\
& =0_{\mathcal{F}}^{!}[\mathcal{A}]-\sum_{i \geq 0}(-1)^{i} 0_{\mathcal{F}}^{!}\left[R^{i} \tau_{*} f_{*} f^{*} \tau^{*} \mathcal{A}\right] \in K_{0}(X) .
\end{aligned}
$$

Definition 4.3. The cosection localized Gysin map

$$
0_{\mathcal{F}, \sigma}^{!}: \underline{\operatorname{Coh}}_{\sigma}(\mathcal{F}) \longrightarrow K_{0}(X(\sigma))
$$

for $\mathcal{F}$ is defined by

$$
0_{\mathcal{F}, \sigma}^{!}[\mathcal{A}]=\left(\left.\tau\right|_{D}\right)_{*}\left(D^{\vee} \cdot 0_{\mathcal{F}^{\prime}}^{!}\left[f^{*} \tau^{*} \mathcal{A}\right]\right)+0_{\left.\mathcal{F}\right|_{X(\sigma)}}^{!} R_{\mathcal{A}} \in K_{0}(X(\sigma))
$$

for any coherent sheaf $\mathcal{A}$ on $\mathcal{F}$ with support in $\mathcal{F}(\sigma)$.

By adding (4.13) and (4.16), we obtain the following comparison of the Gysin maps $0 !$ and $0 !$

Proposition 4.4. For $[\mathcal{A}] \in \underline{\operatorname{Coh}}_{\sigma}(\mathcal{F})$, we have the equality

$$
\iota_{*} 0_{\mathcal{F}, \sigma}(\mathcal{A})=0_{\mathcal{F}}^{!}[\mathcal{A}] \in K_{0}(X) .
$$

\subsection{Cosection localized virtual structure sheaf for almost perfect obstruction theory}

Using (4.8), (4.9) and (4.17), we may now generalize the cosection localized virtual structure sheaf in [14] to Deligne-Mumford stacks equipped with almost perfect obstruction theories.

Definition 4.5. The cosection localized virtual structure sheaf for an almost prefect obstruction theory $\phi$ is defined by

$$
\left[\mathcal{O}_{X, \mathrm{loc}}^{\mathrm{vir}}\right]:=\sum_{j \geq 0} 0_{\mathcal{O}_{b_{X}, \sigma}}\left(\mathcal{A}_{j}\right) \in K_{0}(X(\sigma)),
$$

where $0_{\mathcal{O} b_{X}, \sigma}^{!}$is the cosection localized Gysin map in Definition 4.17 and the sheaves $\mathcal{A}_{j}$ are defined in (4.8).

Proposition 4.6. The pushforward of $\left[\sigma_{X, \mathrm{loc}}^{\mathrm{vir}}\right]$ by the inclusion $\imath: X(\sigma) \rightarrow X$ is the ordinary virtual structure sheaf $\left[\mathrm{O}_{X}^{\mathrm{vir}}\right] \in K_{0}(X)$ in [16].

Proof. By Proposition 4.4, (4.9) and Definition 2.11, we have

$$
\begin{gathered}
l_{*}\left[\mathcal{G}_{X, \mathrm{loc}}^{\mathrm{vir}}\right]=\sum_{j \geq 0} l_{*} 0_{\mathcal{O} b_{X}, \sigma}\left(\mathcal{A}_{j}\right)=\sum_{j \geq 0} 0_{\mathcal{O}_{b_{X}}}^{!}\left[\mathcal{A}_{j}\right] \\
=0_{\mathcal{O} b_{X}} \sum_{j \geq 0}\left[\mathcal{A}_{j}\right]=0_{\mathcal{O} b_{X}}^{!}\left[\mathcal{O}_{\mathfrak{c}_{X / S}}\right]=\left[\mathcal{O}_{X}^{\mathrm{vir}}\right]
\end{gathered}
$$

as desired. 
The cosection localized virtual structure sheaf is deformation invariant. The proof is rather standard and can be found in the Appendix.

Remark 4.7. As discussed carefully in [14], we can be quite flexible in choosing a lift of $\mathcal{A}$ to a class in $K_{0}\left(\mathcal{F}^{\prime}\right)$. Above, we used (4.12) for simplicity, but we could use left derived pullbacks $L f^{*}$ and $L \tau^{*}$ instead of the ordinary pullbacks $f^{*}$ and $\tau^{*}$. With this derived choice, we have a homomorphism

$$
0_{\mathcal{F}, \text { loc }}^{!}: K_{0}(\mathcal{F}(\sigma)) \longrightarrow K_{0}(X(\sigma))
$$

where $K_{0}(\mathcal{F}(\sigma))$ is the Grothendieck group of the abelian category $\operatorname{Coh}_{\sigma}(\mathcal{F})$ of coherent sheaves on $\mathcal{F}$ with support in $\mathcal{F}(\sigma)$.

\section{Virtual torus localization}

A virtual torus localization formula has been established at the level of intersection theory for virtual fundamental cycles in the cases of perfect [7] and semi-perfect obstruction theory [11] and at the level of $K$-theory for virtual structure sheaves for perfect obstruction theory [26]. In this section, we generalize the formula to the setting of virtual structure sheaves in $K$-theory obtained by an almost perfect obstruction theory.

\subsection{T-equivariant almost perfect obstruction theory}

Let $T=\mathbb{C}^{*}$ denote the one-dimensional torus and $X$ a Deligne-Mumford stack with an action of $T$. We denote the fixed locus by $F$. This is the closed substack locally defined by $\operatorname{Spec} A /\left(A^{m v}\right)$ on an equivariant étale chart $\operatorname{Spec} A \rightarrow X$, where $\left(A^{m v}\right)$ denotes the ideal generated by weight spaces corresponding to non-zero $T$-weights. Finally, let

$$
\iota: F \longrightarrow X
$$

denote the inclusion map. For details on group actions on stacks, we refer the reader to [27].

We can give the following definition, which generalizes directly the definition of an almost perfect obstruction theory.

Definition 5.1. ( $T$-equivariant almost perfect obstruction theory) Let $X$ be a Deligne-Mumford stack with a $T$-action. A $T$-equivariant almost perfect obstruction theory $\phi$ consists of the following data:

(a) A $T$-equivariant étale covering $\left\{X_{\alpha} \rightarrow X\right\}_{\alpha \in A}$ of $X$

(b) For each index $\alpha \in A$, an object $E_{\alpha} \in D\left(\left[X_{\alpha} / T\right]\right)$ and a morphism $\phi_{\alpha}: E_{\alpha} \rightarrow \mathbb{L}_{X_{\alpha}}$ in $D\left(\left[X_{\alpha} / T\right]\right)$ that is a perfect obstruction theory on $X_{\alpha}$

These are required to satisfy the following conditions:

1. For each pair of indices $\alpha, \beta$, there exists a $T$-equivariant isomorphism

$$
\psi_{\alpha \beta}:\left.\left.\mathcal{O} b_{X_{\alpha}}\right|_{\alpha \beta} \longrightarrow \mathcal{O} b_{X_{\beta}}\right|_{X_{\alpha \beta}}
$$

so that the collection $\left\{\mathcal{O} b_{X_{\alpha}}=h^{1}\left(E_{\alpha}^{\vee}\right), \psi_{\alpha \beta}\right\}$ gives a descent datum of a sheaf $\mathcal{O} b_{X}$, called the obstruction sheaf, on $X$.

2. For each pair of indices $\alpha, \beta$, there exists a $T$-equivariant étale covering $\left\{V_{\lambda} \rightarrow X_{\alpha \beta}\right\}_{\lambda \in \Gamma}$ of $X_{\alpha \beta}=$ $X_{\alpha} \times_{X} X_{\beta}$ such that for any $\lambda$, the perfect obstruction theories $\left.\phi_{\alpha}\right|_{V_{\lambda}}$ and $\left.\phi_{\beta}\right|_{V_{\lambda}}$ are isomorphic and compatible with $\psi_{\alpha \beta}$. This means there exists an isomorphism

$$
\eta_{\alpha \beta \lambda}:\left.\left.E_{\alpha}\right|_{V_{\lambda}} \longrightarrow E_{\beta}\right|_{V_{\lambda}}
$$


in $D\left(\left[V_{\lambda} / T\right]\right)$ fitting in a commutative diagram

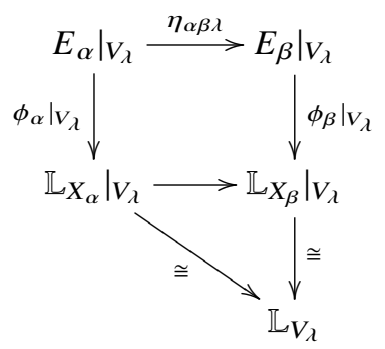

which moreover satisfies $h^{1}\left(\eta_{\alpha \beta \lambda}^{\vee}\right)=\left.\psi_{\alpha \beta}^{-1}\right|_{V_{\lambda}}$.

In the above, $D\left(\left[X_{\alpha} / T\right]\right)$ and $D\left(\left[V_{\lambda} / T\right]\right)$ denote the bounded derived categories of $T$-equivariant quasi-coherent sheaves on $U_{\alpha}$ and $V_{\lambda}$, respectively.

Remark 5.2. When the étale covering in part (a) of the definition consists of the identity morphism id: $X \rightarrow X$, part (b) gives a $T$-equivariant perfect obstruction theory on $X$, and conditions $1 \& 2$ are vacuously satisfied. Thus a $T$-equivariant perfect obstruction theory on $X$ is a special case of a $T$-equivariant almost perfect obstruction theory.

\subsection{T-equivariant almost perfect obstruction theory on the fixed locus}

Suppose that $X$ is a Deligne-Mumford stack with an action of $T$, equipped with a $T$-equivariant almost perfect obstruction theory as above. Let $F_{\alpha}=X_{\alpha} \times_{X} F$, so that $\left\{F_{\alpha} \rightarrow F\right\}_{\alpha \in A}$ gives an étale covering of the fixed locus $F$.

For each index $\alpha$, we have the decomposition

$$
\left.E_{\alpha}\right|_{F_{\alpha}}=\left.\left.E_{\alpha}\right|_{F_{\alpha}} ^{f i x} \oplus E_{\alpha}\right|_{F_{\alpha}} ^{m v}
$$

into the $T$-fixed and moving part. Moreover, $\left.\phi_{\alpha}\right|_{F_{\alpha}}$ similarly decomposes as a direct sum of

$$
\phi_{\alpha}^{f i x}:\left.\left.E_{\alpha}\right|_{F_{\alpha}} ^{f i x} \rightarrow \mathbb{L}_{X_{\alpha}}\right|_{F_{\alpha}} ^{f i x} \text { and } \phi_{\alpha}^{m v}:\left.\left.E_{\alpha}\right|_{F_{\alpha}} ^{m v} \rightarrow \mathbb{L}_{X_{\alpha}}\right|_{F_{\alpha}} ^{m v}
$$

Since $F_{\alpha}$ has a trivial $T$-action, the morphism $\left.\mathbb{L}_{X_{\alpha}}\right|_{F_{\alpha}} \rightarrow \mathbb{L}_{F_{\alpha}}$ factors through

$$
\left.\mathbb{L}_{X_{\alpha}}\right|_{F_{\alpha}} ^{f i x} \longrightarrow \mathbb{L}_{F_{\alpha}}
$$

Composing (5.3) and (5.4), we obtain a morphism

$$
\phi_{\alpha}^{F}:\left.E_{\alpha}\right|_{F_{\alpha}} ^{f i x} \longrightarrow \mathbb{L}_{F_{\alpha}}
$$

By [7], this gives a perfect obstruction theory on $F_{\alpha}$.

Proposition 5.3. The étale covering $\left\{F_{\alpha} \rightarrow F\right\}$ and the perfect obstruction theories $\phi_{\alpha}^{F}:\left.E_{\alpha}\right|_{F_{\alpha}} ^{f i x} \rightarrow \mathbb{L}_{F_{\alpha}}$ form an induced almost perfect obstruction theory $\phi^{F}$ on the fixed locus $F$ with obstruction sheaf $\mathcal{O} b_{F}=\left.\mathcal{O} b_{X}\right|_{F} ^{f i x}$.

Proof. We need to verify that conditions (1) and (2) in Definition 2.10 hold for the perfect obstruction theories on the given étale cover of $F$. 
It is clear that $\mathcal{O} b_{F_{\alpha}}=h^{1}\left(\left.E_{\alpha}^{\vee}\right|_{F_{\alpha}} ^{f i x}\right)=\left.\mathcal{O} b_{X_{\alpha}}\right|_{F_{\alpha}} ^{f i x}$. Since $F_{\alpha \beta}=F_{\alpha} \times_{F} F_{\beta}$ is the fixed locus of $X_{\alpha \beta}=X_{\alpha} \times_{X} X_{\beta}$ and $\psi_{\alpha \beta}$ is $T$-equivariant, we obtain induced isomorphisms

$$
\psi_{\alpha \beta}^{F}:=\left.\psi_{\alpha \beta}\right|_{F_{\alpha \beta}} ^{f i x}:\left.\left.\mathcal{O} b_{F_{\alpha}}\right|_{F_{\alpha \beta}} \longrightarrow \mathcal{O} b_{F_{\beta}}\right|_{F_{\alpha \beta}}
$$

that satisfy the cocycle condition and give descent data for the obstruction sheaf $\mathcal{O} b_{F}=\left.\mathcal{O} b_{X}\right|_{F} ^{f i x}$.

Let $V_{\lambda}^{T}$ denote the fixed locus of $V_{\lambda}$. Similarly, by $T$-equivariance, the isomorphisms $\eta_{\alpha \beta \lambda}$ induce isomorphisms

$$
\eta_{\alpha \beta \lambda}^{F}:=\left.\eta_{\alpha \beta \lambda}\right|_{V_{\lambda}^{T}} ^{f i x}:\left.\left.E_{\alpha}\right|_{V_{\lambda}^{T}} ^{f i x} \longrightarrow E_{\beta}\right|_{V_{\lambda}^{T}} ^{f i x}
$$

fitting in a commutative diagram

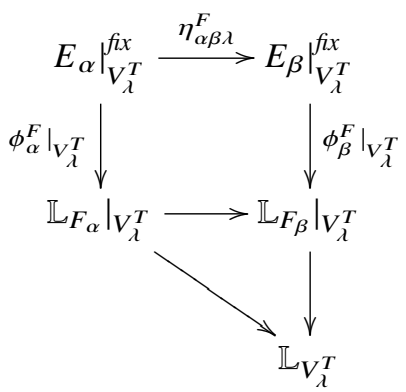

and satisfying $h^{1}\left(\left.\eta_{\alpha \beta \lambda}^{\vee}\right|_{V_{\lambda}^{T}} ^{f i x}\right)=\left.\psi_{\alpha \beta}^{-1}\right|_{F_{\alpha \beta}} ^{f i x}$, as desired.

Let $N_{\alpha}^{\mathrm{vir}}=\left(\left.E_{\alpha}\right|_{F_{\alpha}} ^{m v}\right)^{\vee}$ be the virtual normal bundle of $F_{\alpha}$ in $X_{\alpha}$, and write $E_{\alpha}^{F}=\left.E_{\alpha}\right|_{F_{\alpha}} ^{f i x}$ for brevity from now on.

To prove the torus localization formula in the next subsection, we will need to modify the almost perfect obstruction theory $\phi^{F}$ on $F$. To this end, we make the following assumption.

Assumption 5.4. There exists a two-term complex

$$
N^{\mathrm{vir}}=\left[N_{0} \longrightarrow N_{1}\right]
$$

of locally free sheaves on $F$ and an isomorphism $\mu:\left.h^{1}\left(N^{\mathrm{vir}}\right) \rightarrow \mathcal{O} b_{\phi}\right|_{F} ^{m v}$ such that for any index $\alpha$, we have an isomorphism $\left.N^{\mathrm{vir}}\right|_{F_{\alpha}} \cong N_{\alpha}^{\mathrm{vir}}$ whose homology in degree 1 induces the restriction $\left.\mu\right|_{F_{\alpha}}$. We write $N^{-1}=N_{1}^{\vee}, N^{0}=N_{0}^{\vee}$.

This assumption may turn out to be unnecessary in the future, but under the current state of technology, this is a weakest assumption for a proof of the virtual torus localization formula, Theorem 5.15 below.

We will compare the virtual structure sheaves $\left[\mathcal{O}_{X}^{\text {vir }}\right]$ on $X$ and $\left[\mathcal{O}_{F}^{\text {vir }}\right]$ on $F$ through an intermediate virtual structure sheaf $\left[\widetilde{\mathcal{O}}_{F}^{\mathrm{vir}}\right]$, after introducing an auxiliary almost perfect obstruction theory on $F$. Here is an outline:

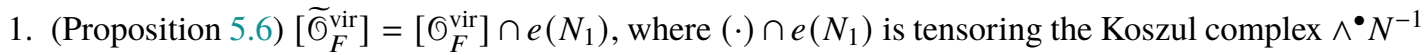
for the zero section of $N_{1}$.

2. (Proposition 5.9) $\imath^{!}\left[\mathcal{O}_{X}^{\mathrm{vir}}\right]=\left[\widetilde{\widetilde{O}}_{F}^{\mathrm{vir}}\right]$, where $\imath: F \rightarrow X$ is the inclusion and $\imath^{!}$is the virtual pullback defined in [26].

3. (Proposition 5.13) $\iota_{*} \xi=\left[\mathcal{O}_{X}^{\mathrm{vir}}\right]$ for some $\xi \in K_{0}(F) \otimes \mathbb{Q} \mathbb{Q}(t)$.

4. (Theorem 5.15) Since $\xi \cap e\left(N_{0}\right)=l^{!} l_{*} \xi=l^{!}\left[\mathcal{O}_{X}^{\mathrm{vir}}\right]=\left[\mathcal{O}_{F}^{\mathrm{vir}}\right] \cap e\left(N_{1}\right)$, we have $\xi=\left[\mathcal{O}_{F}^{\mathrm{vir}}\right] / e\left(N^{\mathrm{vir}}\right)$, where $e\left(N^{\mathrm{vir}}\right)=e\left(N_{0}\right) / e\left(N_{1}\right)$. We thus obtain the torus localization formula

$$
\left[\circlearrowleft_{X}^{\mathrm{vir}}\right]=\iota_{*} \frac{\left[\circlearrowleft_{F}^{\mathrm{vir}}\right]}{e\left(N^{\mathrm{vir}}\right)}
$$

In the subsequent subsections, we will work out the details of the outline. 


\subsection{An auxiliary almost perfect obstruction theory on the fixed locus}

We will introduce a new almost perfect obstruction theory on $F$ by adding the locally free sheaf $N_{1}$ to the obstruction sheaf and compare the virtual structure sheaves arising from the old and new almost perfect obstruction theories.

For each index $\alpha$, we let

$$
\widetilde{E}_{\alpha}^{F}=\left.E_{\alpha}^{F} \oplus N_{1}^{\vee}\right|_{F_{\alpha}}[1] \text { and } \widetilde{\phi}_{\alpha}^{F}: \widetilde{E}_{\alpha}^{F} \longrightarrow E_{\alpha}^{F} \stackrel{\phi_{\alpha}^{F}}{\longrightarrow} \mathbb{L}_{F_{\alpha}}
$$

where the first arrow $\widetilde{E}_{\alpha}^{F} \rightarrow E_{\alpha}^{F}$ in the composition is projection onto the first summand. It is clear that $\widetilde{\phi}_{\alpha}^{F}$ is a perfect obstruction theory on $F_{\alpha}$ with obstruction sheaf $\mathcal{O} b_{\widetilde{\phi}_{\alpha}^{F}}=h^{1}\left(\left(\widetilde{E}_{\alpha}^{F}\right)^{\vee}\right)=\left.\mathcal{O} b_{\phi_{\alpha}^{F}} \oplus N_{1}\right|_{F_{\alpha}}$.

Proposition 5.5. The étale covering $\left\{F_{\alpha} \rightarrow F\right\}$ and the perfect obstruction theories $\widetilde{\phi}_{\alpha}^{F}: \widetilde{E}_{\alpha}^{F} \rightarrow \mathbb{L}_{F_{\alpha}}$ form an almost perfect obstruction theory $\widetilde{\phi}^{F}$ on $F$ with obstruction sheaf $\widetilde{\mathcal{O b}}_{F}=\mathcal{O b}_{F} \oplus N_{1}$.

Proof. The proof is identical to that of Proposition 5.3 using

$$
\widetilde{\psi}_{\alpha \beta}^{F}=\psi_{\alpha \beta}^{F} \oplus \operatorname{id}_{\left.N_{1}\right|_{F_{\alpha \beta}}} \quad \text { and } \quad \widetilde{\eta}_{\alpha \beta \lambda}^{F}=\eta_{\alpha \beta \lambda}^{F} \oplus \operatorname{id}_{\left.N_{1}\right|_{V_{\lambda}} ^{T}}
$$

for the appropriate compatibilities.

The two almost perfect obstruction theories $\phi^{F}$ and $\widetilde{\phi}^{F}$ on $F$ induce virtual structure sheaves $\left[\mathcal{O}_{F}^{\mathrm{vir}}\right] \in K_{0}(F)$ and $\left[\widetilde{\sigma}_{F}^{\mathrm{vir}}\right] \in K_{0}(F)$, respectively. These are related by the following formula.

Proposition 5.6. $\left[\widetilde{\mathcal{O}}_{F}^{\mathrm{vir}}\right]=\left[\mathcal{O}_{F}^{\mathrm{vir}}\right] \cap e\left(N_{1}\right) \in K_{0}(F)$ where $(\cdot) \cap e\left(N_{1}\right)$ denotes tensoring with the Koszul complex $\wedge^{\bullet}\left(N^{-1}\right)$ for the zero section of $N_{1}$.

Proof. Let $j_{\phi^{F}}: \mathfrak{c}_{F} \rightarrow \mathcal{O} b_{F}$ be the induced embedding of the coarse intrinsic normal cone stack of $F$ into the obstruction sheaf stack of $\phi^{F}$. By the definition of $\widetilde{\phi}^{F}$, it is easy to see that the embedding $j_{\widetilde{\phi}^{F}}$ is the composition of $j_{\phi^{F}}$ with the inclusion $\mathcal{O} b_{F} \rightarrow{\widetilde{\mathcal{O}} b_{F}}=\mathcal{O} b_{F} \oplus N_{1}$ as the first summand.

By Definition 2.11, we have

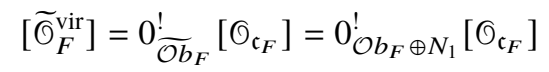

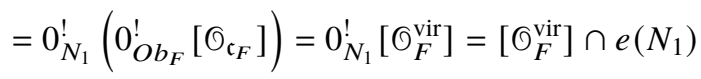

as desired.

\subsection{Refined intersection with the fixed locus}

In this subsection, we prove Proposition 5.9 below.

Lemma 5.7. $\left[\widetilde{\mathcal{O}}_{F}^{\mathrm{vir}}\right]=0_{\left.\mathcal{O} b_{X}\right|_{F} \oplus N_{0}}\left[\mathcal{O}_{\mathfrak{c}_{F / \mathcal{C}_{X}}}\right] \in K_{0}(F)$.

Proof. The proof is an adaptation of a standard functoriality argument, following the lines of the proof of [16, Theorem 4.3]. We repeat a sketch of the argument here for the convenience of the reader.

Let $\mathcal{M}_{X}^{\circ} \rightarrow \mathbb{P}^{1}$ be the deformation of Spec $\mathbb{C}$ to the intrinsic normal cone stack $\mathcal{C}_{X}$. Let $\mathcal{W}=$ $\mathcal{M}_{F \times \mathbb{P}^{1} / \mathcal{M}_{X}^{\circ}}^{\circ}$ be the double deformation space given by the deformation of $F \times \mathbb{P}^{1}$ inside $\mathcal{M}_{X}^{\circ}$ to its

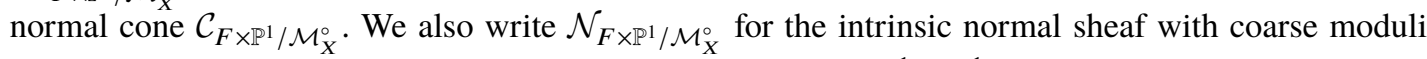

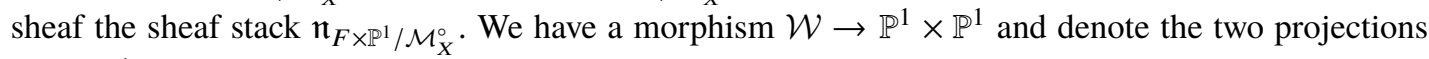
$\mathcal{W} \rightarrow \mathbb{P}^{1}$ by $\pi_{1}$ and $\pi_{2}$, respectively. 
The fiber over $(1,0)$ is $\mathcal{C}_{F}$, while the flat specialization at the point $(0,0)$ along $\{0\} \times \mathbb{P}^{1}$ is $\mathcal{C}_{F / \mathcal{C}_{X}}$. In particular, the flat specialization at $(0,0)$ along $\mathbb{P}^{1} \times\{0\}$ is also $\mathcal{C}_{F / \mathcal{C}_{X}}$, meaning there exists a closed

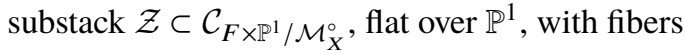

$$
\mathcal{Z}_{t}= \begin{cases}\mathcal{C}_{F}, & t \neq 0 \\ \mathcal{C}_{F / \mathcal{C}_{X},} & t=0\end{cases}
$$

Thus

$$
\begin{aligned}
& {\left[\circlearrowleft_{\mathcal{Z}} \otimes_{\mathscr{O}_{\mathbb{P} 1}}^{L} \mathbb{C}_{0}\right]=\left[\mathcal{G}_{\mathcal{Z}_{0}}\right]=\left[\sigma_{\mathcal{C}_{F / \mathcal{C}_{X}}}\right]} \\
& {\left[\mathcal{O}_{\mathcal{Z}} \otimes_{\mathscr{O}_{\mathbb{P} 1}}^{L} \mathbb{C}_{1}\right]=\left[\mathcal{O}_{\mathcal{Z}_{1}}\right]=\left[\mathcal{O}_{\mathcal{C}_{F}}\right] \text {, }}
\end{aligned}
$$

and since $\left[\mathbb{C}_{0}\right]=\left[\mathbb{C}_{1}\right] \in K_{0}\left(\mathbb{P}^{1}\right)$, we obtain

$$
\left[\mathcal{O}_{\mathcal{C}_{F}}\right]=\left[\mathcal{O}_{\mathcal{C}_{F / \mathcal{C}_{X}}}\right] \in K_{0}\left(\mathcal{C}_{F \times \mathbb{P}^{1} / \mathcal{M}_{X}^{\circ}}\right)
$$

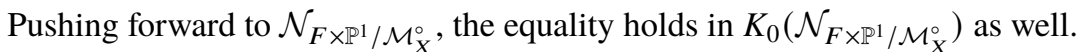

The same argument at the level of coarse moduli sheaves yields the equality

$$
\left[\mathcal{O}_{\mathfrak{c}_{F}}\right]=\left[\mathcal{O}_{\mathfrak{c}_{F / \mathcal{C}_{X}}}\right] \in K_{0}\left(\mathfrak{n}_{\left.F \times \mathbb{P}^{1} / \mathcal{M}_{X}^{\circ}\right)}\right.
$$

As in, [17], for each $\alpha$ we have a commutative diagram of exact triangles on $F_{\alpha} \times \mathbb{P}^{1}$

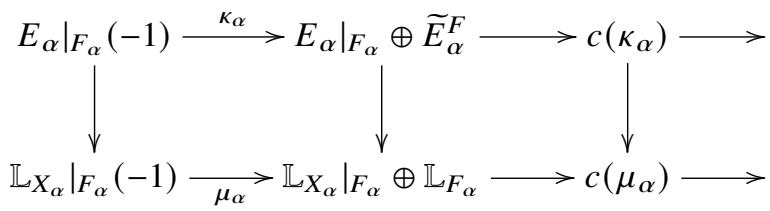

where $\kappa_{\alpha}=\left(z_{0} \cdot \mathrm{id}, z_{1} \cdot g_{\alpha}\right)$ with $z_{0}, z_{1}$ homogeneous coordinates on $\mathbb{P}^{1}$, and $g_{\alpha}$ is the canonical morphism from the inclusion $F_{\alpha} \rightarrow U_{\alpha}$. The morphism $\mu_{\alpha}$ is the restriction to $F_{\alpha}$ of a global arrow $\mu$,

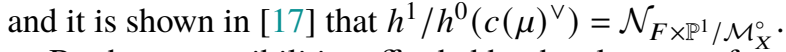

By the compatibilities afforded by the almost perfect obstruction theories and the definition (5.9) of $\widetilde{E}_{\alpha}^{F}$, we see that the closed embeddings

$$
h^{1}\left(c\left(\mu_{\alpha}\right)^{\vee}\right) \longrightarrow h^{1}\left(c\left(\kappa_{\alpha}\right)^{\vee}\right)
$$

glue to a global embedding of sheaf stacks on $X \times \mathbb{P}^{1}$

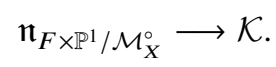

Moreover, it is routine to check that the fiber of $\mathcal{K}$ over $0 \in \mathbb{P}^{1}$ is $\left.\mathcal{O} b_{X}\right|_{F} \oplus N_{0}$, while the fiber at $1 \in \mathbb{P}^{1}$ is $\widetilde{\mathcal{O b}}_{F}$. Therefore, (5.11) and the discussion preceding it imply that

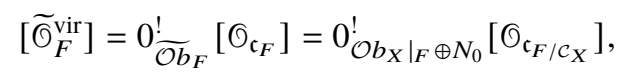

as desired. 
By the definition of $\widetilde{E}_{\alpha}^{F}$, for any index $\alpha$, we have a commutative diagram of exact triangles

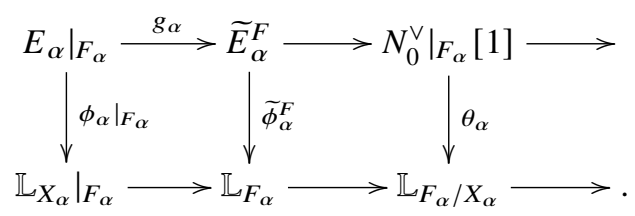

By the following proposition, $\theta_{\alpha}$ gives a perfect obstruction theory on the morphism $F_{\alpha} \rightarrow U_{\alpha}$.

Proposition 5.8. The étale covering $\left\{F_{\alpha} \rightarrow F\right\}$ and the perfect obstruction theories $\theta_{\alpha}:\left.N_{0}^{\vee}\right|_{F_{\alpha}}[1] \rightarrow$ $\mathbb{L}_{F_{\alpha} / X_{\alpha}}$ form an almost perfect obstruction theory $\theta$ on $\iota: F \rightarrow X$ with obstruction sheaf $\mathcal{O} b_{F / X}=N_{0}$.

Proof. Since $F_{\alpha} \rightarrow X_{\alpha}$ is a closed embedding, $\mathbb{L}_{F_{\alpha} / X_{\alpha}}$ is supported in degree -1 . The long exact sequence in cohomology for the diagram (5.12) yields

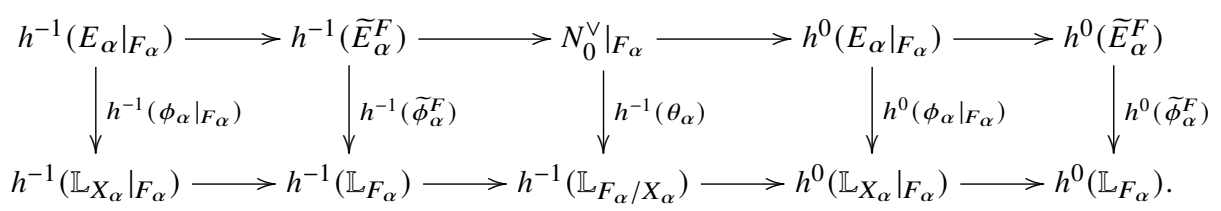

The two leftmost vertical arrows are surjections, while the two rightmost arrows are isomorphisms. Thus, by the five lemma, the middle arrow $h^{-1}\left(\theta_{\alpha}\right)$ is a surjection. Since $h^{0}\left(\left.N_{0}^{\vee}\right|_{F_{\alpha}}[1]\right)=h^{0}\left(\mathbb{L}_{F_{\alpha} / U_{\alpha}}\right)=$ $0, \theta_{\alpha}$ is a perfect obstruction theory for the inclusion $F_{\alpha} \rightarrow X_{\alpha}$.

The rest of the proof is a diagram chase using the definition (5.9) of $\widetilde{E}_{\alpha}^{F}$ and the compatibilities $\psi_{\alpha \beta}$ from Definition 2.10 and $\widetilde{\eta}_{\alpha \beta \lambda}^{F}$ from Proposition 5.5. The details are omitted.

The almost perfect obstruction theory $\theta$ induces a closed embedding

$$
j: \mathfrak{c}_{F / X} \longrightarrow \mathcal{O} b_{F / X}=N_{0}
$$

Since $\iota: F \rightarrow X$ is an embedding, the coarse intrinsic normal cone stack $\mathfrak{c}_{F / X}$ coincides with the intrinsic normal cone $\mathcal{C}_{F / X}$, and we obtain a virtual pullback by the formula

$$
l^{!}: K_{0}(X) \stackrel{\sigma_{l}}{\longrightarrow} K_{0}\left(\mathcal{C}_{F / X}\right) \stackrel{j_{*}}{\longrightarrow} K_{0}\left(N_{0}\right) \stackrel{0_{N_{0}}^{!}}{\longrightarrow} K_{0}(F),
$$

where $\sigma_{l}$ is the deformation to the normal cone (see also [26, §2.1]).

Now we can prove the following.

Proposition 5.9. With the notation above, we have

$$
!\left[\sigma_{X}^{\mathrm{vir}}\right]=\left[\sigma_{F}^{\mathrm{vir}}\right] \cap e\left(N_{1}\right) .
$$

Proof. By Proposition 5.6 and Lemma 5.7, it suffices to prove

$$
l^{!}\left[\mathcal{O}_{X}^{\mathrm{vir}}\right]=0_{\left.\mathcal{O} b_{X}\right|_{F} \oplus N_{0}}\left[\mathcal{O}_{\mathfrak{c}_{F / \mathcal{C}_{X}}}\right] \in K_{0}(F) .
$$

For each local chart $Q=\left(U, \rho, E, r_{E}\right)$ of $O b_{X}$, let $F_{U}=F \times_{X} U, C=\left.\mathfrak{c}_{X}\right|_{U} \times_{\left.O b_{X}\right|_{U}} E$ be the lift of the normal cone $\mathfrak{c}_{X}$ to $E$.

For a closed immersion $Z \hookrightarrow W, M_{Z / W}^{\circ}$ denotes the deformation to the normal cone: that is, it is the blowup of $W \times \mathbb{P}^{1}$ along $Z \times\{0\}$ with the strict transform of $W \times\{0\}$ deleted. We have a flat morphism $M_{Z / W}^{\circ} \rightarrow \mathbb{P}^{1}$ whose fiber over $t \neq 0$ (respectively, $t=0$ ) is $W$ (respectively, the normal cone $C_{Z / W}$ ). 
Then we have a commutative diagram

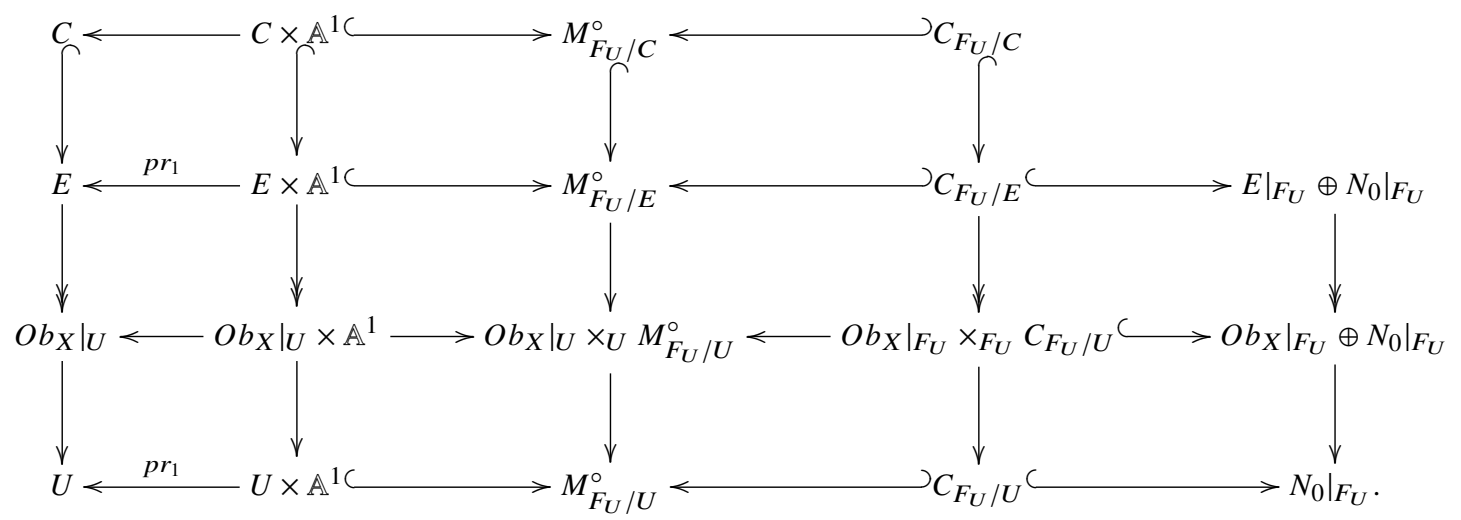

By descent, we have a diagram
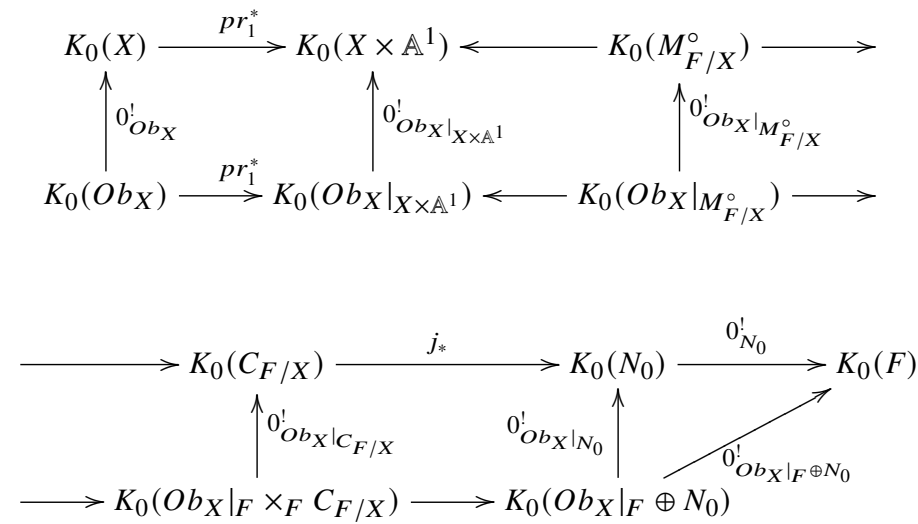

that is commutative by Lemmas 3.7,3.8 and 3.9. Although there are left arrows in (5.15), the composition of the horizontal arrows for the top row is well defined. For the bottom row, the composition of the horizontal arrows evaluated at $\left[\mathcal{O}_{\mathfrak{c}_{X}}\right]$ is well defined and equal to $\left[\mathcal{O}_{\mathfrak{c}_{F / \mathcal{C}_{X}}}\right]$ by (5.14). By the definition of $\imath^{!}, 0_{O b_{X}}^{!}\left[\sigma_{\mathfrak{c}_{X}}\right]=\left[\mathcal{O}_{X}^{\mathrm{vir}}\right]$ is mapped to $\iota^{!}\left[\mathcal{O}_{X}^{\mathrm{vir}}\right]$. Hence (5.13) follows from (5.15).

Finally, the following standard equality for virtual pullbacks holds in our setting (see also [26, Proposition 2.14]).

Proposition 5.10. For any $\mathcal{A} \in K_{0}(F), \iota^{!} l_{*}(\mathcal{A})=\mathcal{A} \cap e\left(N_{0}\right)=\mathcal{A} \otimes \wedge^{\bullet}\left(N^{0}\right)$.

Proof. This is an easy computation, similar to the proof of Proposition 5.6 (but simpler), using the fact that $\sigma_{\iota}(\mathcal{A})=\mathcal{A} \in K_{0}\left(\mathcal{C}_{F / X}\right)$.

\subsection{Virtual torus localization formula}

We are now ready to prove the virtual torus localization formula, using the results of the previous subsections.

All $K$-groups are now considered with $\mathbb{Q}$-coefficients. We denote by $t$ the equivariant parameter so that $K_{0}^{T}(\operatorname{Spec} \mathbb{C})=\mathbb{Q}\left[t, t^{-1}\right]$.

We introduce some terminology. 
Definition 5.11. Let $X$ be a Deligne-Mumford stack with an action of $T$ and $\imath: F \rightarrow X$ denote its $T$-fixed locus. We say that $X$ is admissible for torus localization if the homomorphism

$$
l_{*}: K_{0}^{T}(F) \rightarrow K_{0}^{T}(X)
$$

of $K_{0}^{T}(\operatorname{Spec} \mathbb{C})=\mathbb{Q}\left[t, t^{-1}\right]$-modules becomes an isomorphism after tensoring with $\mathbb{Q}(t)$.

Remark 5.12. By [6], if $X$ is an algebraic space with a $T$-action, then $X$ is admissible.

The following proposition implies that when the $X_{\alpha}$ in condition (a) of Definition 5.1 are schemes, then a Deligne-Mumford stack with a $T$-equivariant almost perfect obstruction theory is admissible.

Proposition 5.13. Let $X$ be a Deligne-Mumford stack with an action of $T$ and a T-equivariant étale atlas $f: U \rightarrow X$ of finite type. Then $X$ is admissible.

Proof. Let $M=X-F$ and $V=f^{-1}(M)$. Using the existence of the equivariant atlas $f: U \rightarrow X$, we have the excision exact sequence for $K$-theory of Deligne-Mumford stacks (see also [31, Proposition 3.3]), and thus it suffices to show that

$$
K_{*}^{T}(M) \otimes_{\mathbb{Q}\left[t, t^{-1}\right]} \mathbb{Q}(t)=0
$$

or, equivalently,

$$
K_{*}^{T}(M) \otimes_{\mathbb{Q}\left[t, t^{-1}\right]} \mathbb{C}(t)=0,
$$

where $K_{*}^{T}(M)$ is the direct sum of all $T$-equivariant $K$-theory groups.

More generally, we will show that if $M$ is a Deligne-Mumford stack with a $T$-action and $T$-equivariant atlas $f: V \rightarrow M$ with empty fixed locus $M^{T}=\emptyset$, then (5.16) holds. In the rest of the proof, all $K$-groups are considered with $\mathbb{C}$-coefficients.

Let $M_{1} \subset M$ be an open substack such that $f: V_{1}=f^{-1}\left(M_{1}\right) \rightarrow M_{1}$ is étale of (maximal) degree $n$. $M_{1}$ is preserved by the $T$-action. Then, as in [18, Proposition 3.5.5.(iii)], we have that $M_{1}$ is isomorphic to the quotient of the complement of all diagonals in the $n$-fold product $V_{1} \times_{M_{1}} \ldots \times_{M_{1}} V_{1}$ by the action of the symmetric group $S_{n}$. Thus $M_{1}$ is of the form $\left[W_{1} / G_{1}\right]$, where $G_{1}$ is a finite group acting on a scheme $W_{1}$ and the $T$-action is given by an action on $W_{1}$ commuting with the $G_{1}$-action and such that $W_{1}^{T}=\emptyset$.

We write $\operatorname{dim}: R\left(G_{1}\right) \rightarrow \mathbb{C}$ for the morphism induced by mapping every representation to its dimension with kernel the augmentation (maximal) ideal $\mathfrak{m}_{G_{1}}$.

We thus have

$$
K_{*}^{T}\left(M_{1}\right)=K_{*}^{T}\left(\left[W_{1} / G_{1}\right]\right)=K_{*}^{T \times G_{1}}\left(W_{1}\right) .
$$

Since $W_{1}^{T}=\emptyset, T$ acts on $W_{1}$ with finite stabilizers, and thus we may choose $h_{1} \in T$ such that $W_{1}^{h_{1}}=\emptyset$. Then $h_{1}$ belongs to the center of $T \times G_{1}$, and so its conjugacy class is just $\left\{h_{1}\right\}$. Therefore, [6, Theorem 3.3(a)] implies that

$$
K_{*}^{T \times G_{1}}\left(W_{1}\right)_{\mathfrak{m}_{h_{1}}}=0,
$$

where $\mathfrak{m}_{h_{1}}$ is the maximal ideal of the complex representation ring $R\left(T \times G_{1}\right)=R\left(G_{1}\right)\left[t, t^{-1}\right]$ generated by the augmentation ideal $\mathrm{m}_{G_{1}}$ of $G_{1}$ and $\left(t-h_{1}\right)$.

In particular, for any element $[x] \in K_{*}^{T \times G_{1}}\left(W_{1}\right)$, there exists a Laurent polynomial $\mu_{1}(t) \in$ $R\left(G_{1}\right)\left[t, t^{-1}\right]-\mathfrak{m}_{h_{1}}$ such that

$$
\mu_{1}(t) \cdot[x]=0 \in K_{*}^{T \times G_{1}}\left(W_{1}\right) .
$$

Letting $\ell_{1}(t)=\mu_{1}(t) \bmod \mathfrak{m}_{G_{1}} \in \mathbb{C}\left[t, t^{-1}\right]$, we have $\ell_{1}(t) \neq 0$ and

$$
\ell_{1}(t) \cdot[x] \in \mathrm{m}_{G_{1}} K_{*}^{T \times G_{1}}\left(W_{1}\right)
$$


so that

$$
\ell_{1}(t) \cdot[x]=\sum m_{i}\left[x_{i}\right]
$$

with $m_{i} \in \mathrm{m}_{G_{1}}$ and $\left[x_{i}\right] \in K_{*}^{T \times G_{1}}\left(W_{1}\right)$.

Repeating the argument for the $\left[x_{i}\right]$, we can find a non-zero Laurent polynomial $\ell_{2}(t) \in \mathbb{C}\left[t, t^{-1}\right]$ such that

$$
\ell_{2}(t) \ell_{1}(t) \cdot[x] \in \mathrm{m}_{G_{1}}^{2} K_{*}^{T \times G_{1}}\left(W_{1}\right)
$$

and, continuing inductively, for any positive integer $N$, non-zero Laurent polynomials $\ell_{1}(t), \ell_{2}(t), \ldots, \ell_{N}(t) \in \mathbb{C}\left[t, t^{-1}\right]$ such that

$$
\ell_{N}(t) \ldots \ell_{1}(t) \cdot[x] \in \mathfrak{m}_{G_{1}}^{N} K_{*}^{T \times G_{1}}\left(W_{1}\right) .
$$

Since $R\left(G_{1}\right)$ is an Artinian $\mathbb{C}$-algebra of finite type, we have $\mathfrak{m}_{G_{1}}^{N}=0$ for large enough $N$. We conclude that for any $[x] \in K_{*}^{T \times G_{1}}\left(W_{1}\right)$, there exists a non-zero Laurent polynomial $\ell(t) \in \mathbb{C}\left[t, t^{-1}\right]$ such that

$$
\ell(t) \cdot[x]=0 \in K_{*}^{T \times G_{1}}\left(W_{1}\right)
$$

which implies that

$$
K_{*}^{T}\left(M_{1}\right) \otimes \mathbb{C}(t)=K_{*}^{T \times G_{1}}\left(W_{1}\right) \otimes \mathbb{C}(t)=0 .
$$

Letting $Z_{1}=M-M_{1}$ be the complement of $M_{1}$, by excision we are reduced to showing that $K_{*}^{T}\left(Z_{1}\right) \otimes \mathbb{C}(t)=0$. Repeating the above argument and using Noetherian induction concludes the proof.

Remark 5.14. By the results of [1], a (separated) Deligne-Mumford stack of finite type with a $T$-action always admits a $T$-equivariant affine étale atlas of finite type after possibly reparameterizing the action of $T$. So assuming the existence of an equivariant atlas is not a restriction for the purposes of this paper.

Theorem 5.15. (Virtual torus localization formula) Let $X$ be an admissible Deligne-Mumford stack with an action of $T$ and a T-equivariant almost perfect obstruction theory $\phi$ such that Assumption 5.4 holds. Let $F$ denote the $T$-fixed locus of $X$ and $\phi^{F}$ its induced almost perfect obstruction theory. Then

$$
\left[\bigcirc_{X}^{\mathrm{vir}}\right]=\iota_{*} \frac{\left[\bigcirc_{F}^{\mathrm{vir}}\right]}{e\left(N^{\mathrm{vir}}\right)} \in K_{0}^{T}(X) \otimes_{\mathbb{Q}\left[t, t^{-1}\right]} \mathbb{Q}(t),
$$

where the Euler class $e\left(N^{\mathrm{vir}}\right)=e\left(N_{0}\right) / e\left(N_{1}\right)=\wedge^{\bullet}\left(\left(N^{\mathrm{vir}}\right)^{\vee}\right)$ denotes multiplication by $\wedge^{\bullet} N^{0} / \wedge^{\bullet} N^{-1}$.

Proof. We first remark that the operation $(\cdot) \cap e\left(N_{0}\right)$ on $K_{0}^{T}(F) \otimes_{\mathbb{Q}\left[t, t^{-1}\right]} \mathbb{Q}(t)$ is invertible. Using the canonical isomorphism $K_{0}^{T}\left(Y^{\text {red }}\right) \cong K_{0}^{T}(Y)$ for any Deligne-Mumford stack $Y$ with a $T$-action (see also [14, Proposition 2.1]), excision for $K$-theory and Noetherian induction, as in the proof of the previous proposition, we may reduce to the case $F=[W / G]$, where $G$ is a finite group acting on a smooth scheme $W$ and the torus $T$ acts trivially on $W$. Then $K_{*}^{T}(F)=K_{*}^{T \times G}(W)$ and [6, Theorem 3.3(b)] applies, choosing any $h \in T$ that is not a root of unity, since the $T$-weights of $N_{0}$ are non-zero and $F$ is the $T$-fixed locus of the total space of the vector bundle $N_{0}$.

Now, by Propositions 5.9 and 5.10, we have

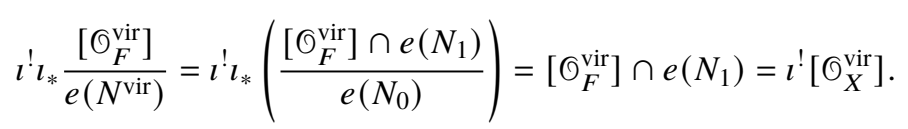

By the previous proposition, $l_{*}$ becomes an isomorphism after tensoring with $\mathbb{Q}(t)$. Hence, Proposition 5.10 implies that $\frac{l^{!}}{e\left(N_{0}\right)}$ is the inverse of $\boldsymbol{l}_{*}$. In particular, $l^{!}$is injective. Thus the virtual torus localization formula follows from (5.17). 


\section{Torus localization of cosection localized virtual structure sheaf}

Torus localization for cosection localized virtual cycles has been established in [5] and [11] in the settings of perfect and semi-perfect obstruction theory, respectively. In this section, we prove the corresponding statement for virtual structure sheaves obtained by an almost perfect obstruction theory. As usual, $T$ denotes the torus $\mathbb{C}^{*}$.

Let $X$ be an admissible Deligne-Mumford stack with a $T$-action and a $T$-equivariant almost perfect obstruction theory $\phi$ given by perfect obstruction theories $\phi_{\alpha}: E_{\alpha} \rightarrow \mathbb{L}_{X_{\alpha}}$ on a $T$-equivariant étale cover $\left\{\rho_{\alpha}: X_{\alpha} \rightarrow X\right\}$. Moreover, suppose that we have a $T$-invariant cosection

$$
\sigma: \mathcal{O} b_{X} \longrightarrow \mathcal{O}_{X}
$$

We use the same notation as in Section 4 and Section 5. Let $F(\sigma)=F \times_{X} X(\sigma)$.

By Proposition 5.3, the fixed locus $F$ admits an almost perfect obstruction theory $\phi^{F}$ on the étale cover $\left\{F_{\alpha} \rightarrow F\right\}$ with obstruction sheaf

$$
\mathcal{O} b_{F}=\left.\mathcal{O} b_{X}\right|_{F} ^{f i x}
$$

Since the cosection $\sigma$ is $T$-invariant, $\left.\sigma\right|_{F}$ factors through a morphism

$$
\sigma_{F}: \mathcal{O} b_{F} \longrightarrow \mathcal{O}_{F}
$$

whose zero locus is precisely $F(\sigma)$. Therefore, by Definition 4.5, we have cosection localized virtual structure sheaves

$$
\left[\circlearrowleft_{X, \mathrm{loc}}^{\mathrm{vir}}\right] \in K_{0}(X(\sigma)), \quad\left[\circlearrowleft_{F, \mathrm{loc}}^{\mathrm{vir}}\right] \in K_{0}(F(\sigma))
$$

Theorem 6.1. Let $\imath: F(\sigma) \rightarrow X(\sigma)$ denote the inclusion, and suppose that Assumption 5.4 holds. Then

$$
\left[\mathcal{\Theta}_{X, \mathrm{loc}}^{\mathrm{vir}}\right]=\iota_{*} \frac{\left[\mathcal{\Theta}_{F, \mathrm{loc}}^{\mathrm{vir}}\right]}{e\left(N^{\mathrm{vir}}\right)} \in K_{0}^{T}(X(\sigma)) \otimes_{\mathbb{Q}\left[t, t^{-1}\right]} \mathbb{Q}(t)
$$

Proof. The proof proceeds along the same steps as the proof of Theorem 5.15, with minor modifications to account for the presence of the cosections $\sigma$ and $\sigma_{F}$.

The proof of Proposition 5.6 goes through, using the cosection

$$
\widetilde{\sigma}_{F}: \widetilde{\mathcal{O}}_{F}=\mathcal{O} b_{F} \oplus N_{1} \longrightarrow \mathcal{O} b_{F} \stackrel{\sigma_{F}}{\longrightarrow} \mathcal{O}_{F}
$$

and working with the sheaves $\mathcal{A}_{j}$ defined in (4.8).

For Lemma 5.7, it is shown in [12, Section 5] that there exists an extended cosection $\bar{\sigma}$ for $\mathcal{K}$ that restricts to the cosection $\widetilde{\sigma}^{F}$ over $1 \in \mathbb{P}^{1}$ and the cosection

$$
\left.\left.\mathcal{O} b_{X}\right|_{F} \oplus N_{0} \longrightarrow \mathcal{O} b_{X}\right|_{F} \stackrel{\left.\sigma\right|_{F}}{\longrightarrow} \mathcal{O}_{F}
$$

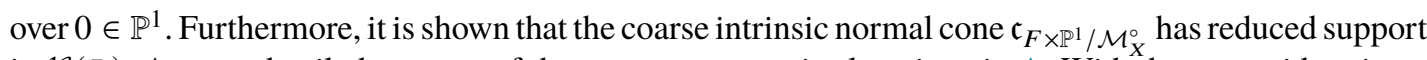
in $\mathcal{K}(\bar{\sigma})$. A more detailed account of the same argument is also given in A. With these considerations, the proof goes through identically.

Finally, in Proposition 5.9, it is easy to check that all the sheaves and cones have the appropriate supports with respect to the cosections at hand. 


\section{Applications}

We now discuss some applications of the theory developed thus far. We establish a wall-crossing formula for simple $\mathbb{C}^{*}$-wall crossing in the setting of almost perfect obstruction theory, using the torus localization formula of Theorem 5.15. Moreover, we show that the Jiang-Thomas dual obstruction cone in [10] admits an almost perfect obstruction theory and thus gives rise to $K$-theoretic invariants, using the combination of torus localization and cosection localization in Theorem 6.1.

\subsection{K-theoretic simple $\mathbb{C}^{*}$-wall crossing}

In this subsection, we establish a $K$-theoretic wall-crossing formula for simple $\mathbb{C}^{*}$-wall crossing, following the construction given in [13].

Let $X$ be an admissible Deligne-Mumford stack acted on by the torus $T=\mathbb{C}^{*}$ and equipped with a $T$-equivariant almost perfect obstruction theory $\phi$ consisting of perfect obstruction theories $\phi_{\alpha}: E_{\alpha} \rightarrow$ $\mathbb{L}_{X_{\alpha}}$ on a $T$-equivariant étale cover $\left\{X_{\alpha} \rightarrow X\right\}$. Let $F$ denote the fixed locus. We assume that Assumption 5.4 holds, so that we have the two-term complex $N^{\text {vir }}=\left[N_{0} \rightarrow N_{1}\right]$ of locally free sheaves on $F$. Let

1. $X^{s}$ be the open substack of $X$, consisting of $x \in X$ such that the orbit $T \cdot x$ is 1-dimensional and closed in $X$;

2. $\Sigma_{ \pm}^{0}=\left\{x \in X-\left(X^{s} \cup F\right) \mid \lim _{t \rightarrow 0} t^{ \pm 1} \cdot x \in F\right\}$;

3. $\Sigma_{ \pm}=\Sigma_{+}^{0} \cup F$;

4. $X_{ \pm}=X-\Sigma_{\mp} \subset X$;

5. $M_{ \pm}=\left[X_{ \pm} / T\right] \subset M=[X / T]$.

We assume that $M_{ \pm}$are separated Deligne-Mumford stacks.

The master space associated with the wall crossing $M_{ \pm}$is defined by

$$
\mathcal{M}=\left[X \times \mathbb{P}^{1}-\Sigma_{-} \times\{0\}-\Sigma_{+} \times\{\infty\} / \mathbb{C}^{*}\right],
$$

where $\mathbb{C}^{*}$ acts trivially on $X$ and on $\mathbb{P}^{1}$ by $t \cdot(a: b)=(a: t b) . \mathcal{M}$ admits an étale cover $\left\{\mathcal{M}_{\alpha} \rightarrow \mathcal{M}\right\}$, where

$$
\mathcal{M}_{\alpha}=\left[X_{\alpha} \times \mathbb{P}^{1}-\left(\Sigma_{-} \times_{X} X_{\alpha}\right) \times\{0\}-\left(\Sigma_{+} \times_{X} X_{\alpha}\right) \times\{\infty\} / \mathbb{C}^{*}\right]
$$

The $T$-action on $X$ induces an action of $T$ on $\mathcal{M}$ with fixed locus

$$
M_{+} \sqcup F \sqcup M_{-} .
$$

For each index $\alpha$, the pullback of $\phi_{\alpha}$ to $X_{\alpha} \times \mathbb{P}^{1}$ is $\mathbb{C}^{*}$-equivariant, and therefore by descent we obtain a morphism $\bar{\phi}_{\alpha}: \bar{E}_{\alpha} \rightarrow \mathbb{L}_{\mathcal{M}_{\alpha}}$.

Proposition 7.1. The morphisms $\bar{\phi}_{\alpha}: \bar{E}_{\alpha} \rightarrow \mathbb{L}_{\mathcal{M}_{\alpha}}$ on the étale cover $\left\{\mathcal{M}_{\alpha} \rightarrow \mathcal{M}\right\}$ form a T-equivariant almost perfect obstruction theory $\bar{\phi}$ on $\mathcal{M}$.

Proof. The proof is straightforward, similar to the arguments given for checking the axioms of almost perfect obstruction theories in Section 5.

Applying the virtual torus localization formula then yields the following theorem.

Theorem 7.2. With the above notation and conditions, we have

$$
\left[\Theta_{M_{+}}^{\mathrm{vir}}\right]-\left[\Theta_{M_{-}}^{\mathrm{vir}}\right]=\operatorname{res}_{t=1} \frac{\left[\Theta_{F}^{\mathrm{vir}}\right]}{e\left(N^{\mathrm{vir}}\right)} \in K_{0}^{T}(\mathcal{M})
$$


Proof. By Theorem 5.15, we have

$$
\left[\mathcal{G}_{\mathcal{M}}^{\mathrm{vir}}\right]=\frac{\left[\mathcal{G}_{M_{+}}^{\mathrm{vir}}\right]}{1-t}+\frac{\left[\mathcal{G}_{M_{-}}^{\mathrm{vir}}\right]}{1-t^{-1}}+\frac{\left[\mathcal{G}_{F}^{\mathrm{vir}}\right]}{e\left(N^{\mathrm{vir}}\right)}
$$

since, by construction, the normal bundle of $M_{+}$is trivial with $T$-weight 1 and the normal bundle of $M_{-}$ is trivial with $T$-weight -1 .

Since $\left[\mathcal{O}_{\mathcal{M}}^{\text {vir }}\right] \in K_{0}^{T}(\mathcal{M})$, it has zero residue at $t=1$. Therefore, taking residues at $t=1$, the left-hand side of (7.2) vanishes, and we get that

$$
-\left[\circlearrowleft_{M_{+}}^{\mathrm{vir}}\right]+\left[\circlearrowleft_{M_{-}}^{\mathrm{vir}}\right]+\operatorname{res}_{t=1} \frac{\left[\sigma_{F}^{\mathrm{vir}}\right]}{e\left(N^{\mathrm{vir}}\right)}
$$

is zero, which is what we want.

\subsection{Dual obstruction cone}

We first recall the definition of the Jiang-Thomas dual obstruction cone [10].

Let $X$ be a Deligne-Mumford stack equipped with a perfect obstruction theory $\phi: E \rightarrow \mathbb{L}_{X}$ with obstruction sheaf $\mathcal{F}=O b_{X}=h^{1}\left(E^{\vee}\right)$.

Definition 7.3. The dual obstruction cone of $X$ is defined by

$$
N=\operatorname{Spec}_{X}(\operatorname{Sym} \mathcal{F}) \stackrel{\pi}{\rightarrow} X,
$$

which is the functor that assigns to every morphism $\rho: U \rightarrow X$ the set $\operatorname{Hom}_{U}\left(\rho^{*} \mathcal{F}, \mathcal{O}_{U}\right)$.

By standard perfect obstruction theory arguments, we can find an étale cover $\left\{X_{\alpha} \rightarrow X\right\}$, a smooth affine scheme $A_{\alpha}$, a vector bundle $\mathcal{V}_{\alpha}$ on $A_{\alpha}$ and a section $s_{\alpha} \in H^{0}\left(A_{\alpha}, \mathcal{V}_{\alpha}\right)$ such that $X_{\alpha}$ is the zero locus of $s_{\alpha}$ and the perfect obstruction theory on $X_{\alpha}$ is given by the two-term complex

$$
E_{\alpha}=\left.E\right|_{X_{\alpha}} \simeq\left[\left.\left.\mathcal{V}_{\alpha}^{\vee}\right|_{X_{\alpha}} \stackrel{d s_{\alpha}^{\vee}}{\longrightarrow} \Omega_{A_{\alpha}}\right|_{X_{\alpha}}\right]
$$

together with the natural map to $\mathbb{L}_{X_{\alpha}} \simeq\left[I_{\alpha} /\left.I_{\alpha}^{2} \rightarrow \Omega_{A_{\alpha}}\right|_{X_{\alpha}}\right]$, where $I_{\alpha}$ is the ideal sheaf of $X_{\alpha}$ in $A_{\alpha}$.

Let $N_{\alpha}=\left.N\right|_{X_{\alpha}}$, and write $\pi_{\alpha}:\left.\mathcal{V}_{\alpha}^{\vee}\right|_{X_{\alpha}} \rightarrow X_{\alpha}$ for the projection. By definition, $N_{\alpha}$ is the closed subscheme of $\left.\mathcal{V}_{\alpha}^{\vee}\right|_{X_{\alpha}}$ defined by the vanishing of the section

$$
d s_{\alpha}^{\vee}:\left.\left.\mathcal{V}_{\alpha}^{\vee}\right|_{X_{\alpha}} \longrightarrow \pi_{\alpha}^{*} \Omega_{A_{\alpha}}\right|_{X_{\alpha}}
$$

Let $x_{1}, \ldots, x_{n}$ be étale coordinates on $A_{\alpha}$ and $y_{1}, \ldots, y_{r}$ coordinates on the fibers of the bundle $\mathcal{V}_{\alpha}^{\vee}$. Then $N_{\alpha}$ is cut out by the equations

$$
\left\{s_{i}\right\}_{1 \leq i \leq r},\left\{\sum_{i} y_{i} \frac{\partial s_{i}}{\partial x_{j}}\right\}_{1 \leq j \leq n},
$$

where $s_{i}=s_{i}\left(x_{1}, \cdots, x_{n}\right)$ are the coordinate functions of the section $s_{\alpha}$.

Let $\widetilde{s}_{\alpha}: \mathcal{V}_{\alpha}^{\vee} \rightarrow \mathbb{C}$ be the function defined by the formula

$$
\widetilde{s}_{\alpha}=\sum_{i} y_{i} s_{i}
$$


The differential of $\widetilde{s}_{\alpha}$ is then

$$
d \widetilde{s}_{\alpha}=\sum_{i} s_{i} d y_{i}+\sum_{j}\left(\sum_{i} y_{i} \frac{\partial s_{i}}{\partial x_{j}}\right) d x_{j} .
$$

Comparing (7.5) with (7.4), we see that $N_{\alpha}$ is the d-critical locus of the function $\widetilde{s}_{\alpha}$. It therefore admits a symmetric perfect obstruction theory (see also [3]) $\psi_{\alpha}: F_{\alpha} \rightarrow \mathbb{L}_{N_{\alpha}}$ with

$$
F_{\alpha}=\left[\left.\left.T_{\mathcal{V}_{\alpha}^{\vee}}\right|_{N_{\alpha}} \stackrel{d\left(d \widetilde{s}_{\alpha}\right)^{\vee}}{\longrightarrow} \Omega_{\mathcal{V}_{\alpha}^{\vee}}\right|_{N_{\alpha}}\right]
$$

and obstruction sheaf $\mathcal{O} b_{N_{\alpha}}=h^{1}\left(F_{\alpha}^{\vee}\right)=\Omega_{N_{\alpha}}$.

Theorem 7.4. The étale cover $\left\{N_{\alpha} \rightarrow N\right\}$ and the (symmetric) perfect obstruction theories $\psi_{\alpha}: F_{\alpha} \rightarrow$ $\mathbb{L}_{N_{\alpha}}$ form an almost perfect obstruction theory $\psi$ on $N$ with obstruction sheaf $\mathcal{O} b_{N}=\Omega_{N}$.

Proof. Let $\pi: N \rightarrow X$ denote the projection. For any index $\alpha$, we have a commutative diagram

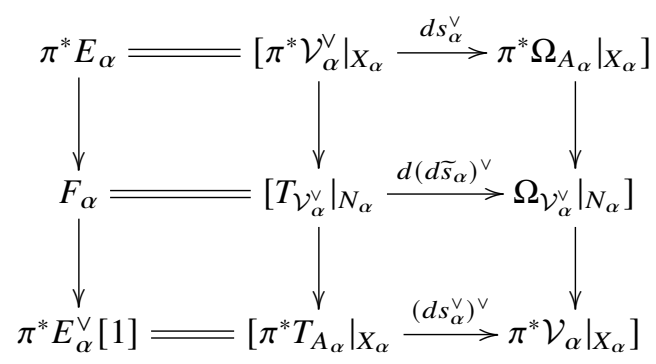

giving an exact triangle $\pi^{*} E_{\alpha} \rightarrow F_{\alpha} \rightarrow \pi^{*} E_{\alpha}^{\vee}[1]$ fitting in a commutative diagram

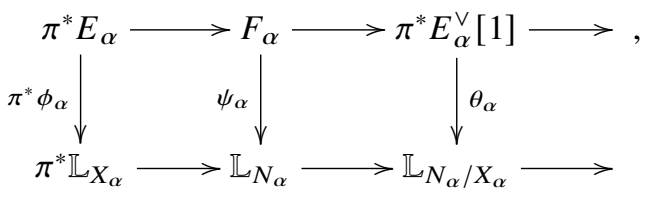

where $\pi^{*} \phi_{\alpha}$ and $\theta_{\alpha}$ are restrictions to $N_{\alpha}$ of global arrows $\pi^{*} \phi: \pi^{*} E \rightarrow \pi^{*} \mathbb{L}_{X}$ and $\theta: \pi^{*} E^{\vee}[1] \rightarrow$ $\pi^{*} \mathcal{F} \rightarrow \mathbb{L}_{N / X}$ using the isomorphisms (7.3) and the definition of the dual obstruction cone $N$. Since $\mathcal{O} b_{N_{\alpha}}=\Omega_{N_{\alpha}}=\left.\Omega_{N}\right|_{N_{\alpha}}$, the obstruction sheaves glue to an obstruction sheaf $\mathcal{O} b_{N}=\Omega_{N}$.

Let $X_{\alpha \beta}=X_{\alpha} \times_{X} X_{\beta}$ and $N_{\alpha \beta}=N_{\alpha} \times_{N} N_{\beta}=N \times_{X} X_{\alpha \beta}$. Then (7.8) together with the above discussion shows that there exist quasi-isomorphisms

$$
\eta_{\alpha \beta}:\left.\left.F_{\alpha}\right|_{N_{\alpha \beta}} \longrightarrow F_{\beta}\right|_{N_{\alpha \beta}}
$$

that are compatible with the morphisms $\left.\psi_{\alpha}\right|_{N_{\alpha \beta}}$ and $\left.\psi_{\beta}\right|_{N_{\alpha \beta}}$ and respect the symmetry of the obstruction theories $F_{\alpha}$ and $F_{\beta}$, thus inducing the gluing morphisms for the obstruction sheaf $\Omega_{N}$. In particular, the axioms of an almost perfect obstruction theory are all satisfied.

Remark 7.5. The almost perfect obstruction theory of $N$ is symmetric in the sense of [3] with respect to the natural generalization of the definition in our context.

From now on, we assume that the perfect obstruction theory $E$ has a global resolution $E=\left[E^{-1} \rightarrow\right.$ $\left.E^{0}\right]$, where $E^{-1}, E^{0}$ are locally free sheaves on $X$.

The grading on $\operatorname{Sym} \mathcal{F}$ determines a $T=\mathbb{C}^{*}$-action on $N$, scaling the fibers of $N$ over $X$, whose fixed locus is precisely $X$. Differentiating the $T$-action, we obtain the Euler vector field whose dual is a 
cosection

$$
\sigma: \Omega_{N} \longrightarrow O_{N}
$$

and whose vanishing locus is $X$ by [10, Section 3].

By construction, the almost perfect obstruction theory $\psi$ of Theorem 7.4 is $T$-equivariant and has obstruction sheaf $\mathcal{O} b_{N}=\Omega_{N}$. By (7.7), the virtual normal bundle of $X$ inside $N$ is $E^{\vee}$, which by assumption admits the global resolution $\left[E_{0} \rightarrow E_{1}\right]$, where as usual, $E_{i}=\left(E^{-i}\right)^{\vee}$ for $i=0$, 1 . Therefore Assumption 5.4 holds, and we may apply Theorem 5.15 and Definition 4.5 to obtain the following theorem, keeping in mind that the cosection $\sigma$ vanishes on the $T$-fixed locus $X$.

Theorem 7.6. Let $X$ be a Deligne-Mumford stack with a perfect obstruction theory $\phi: E \rightarrow \mathbb{L}_{X}$ with obstruction sheaf $\mathcal{F}=\mathcal{O} b_{X}$ such that $E$ admits a global resolution by locally free sheaves. Let $\psi$ denote the induced T-equivariant almost perfect obstruction theory of the dual obstruction cone $N=\operatorname{Spec}_{X}(\operatorname{Sym} F)$ with obstruction sheaf $\mathcal{O} b_{N}=\Omega_{N}$ and cosection $\sigma$, as in (7.9). Let $\iota: X \rightarrow N$ be the inclusion as the zero section. Then the cosection localized virtual structure sheaf $\left[\mathcal{O}_{N, \mathrm{loc}}^{\mathrm{vir}}\right] \in K_{0}(X)$, and the virtual structure sheaves $\left[\mathcal{O}_{N}^{\mathrm{vir}}\right] \in K_{0}(N)$ and $\left[\mathcal{G}_{X}^{\mathrm{vir}}\right] \in K_{0}(X)$ are related by

$$
\begin{aligned}
& {\left[\circlearrowleft_{N}^{\mathrm{vir}}\right]=\iota_{*}\left[\circlearrowleft_{N, \mathrm{loc}}^{\mathrm{vir}}\right] \in K_{0}(N),} \\
& {\left[\circlearrowleft_{N}^{\mathrm{vir}}\right]=\iota_{*} \frac{\left[\circlearrowleft_{X}^{\mathrm{vir}}\right]}{e\left(E^{\vee}\right)} \in K_{0}^{T}(N) \otimes_{\mathbb{Q}\left[t, t^{-1}\right]} \mathbb{Q}(t) .}
\end{aligned}
$$

In particular, when $X$ is proper, we may define $K$-theoretic invariants by taking regular and equivariant Euler characteristics, respectively, to obtain

$$
\chi\left(\left[\mathcal{O}_{N, \mathrm{loc}}^{\mathrm{vir}}\right]\right) \in \mathbb{Q}, \quad \chi_{t}\left(\frac{\left[\mathcal{O}_{X}^{\mathrm{vir}}\right]}{e\left(E^{\vee}\right)}\right) \in \mathbb{Q}(t) .
$$

Remark 7.7. $T$-equivariant $K$-theoretic invariants refining the Jiang-Thomas signed virtual Euler characteristic studied in [10] have also been defined in [30, Section 2.5] for (-1)-shifted cotangent bundles of quasi-smooth derived projective schemes.

\section{A. Deformation invariance of cosection localized virtual structure sheaf}

Let $\phi$ be an almost perfect obstruction theory on $X \rightarrow S$, given by perfect obstruction theories $\phi_{\alpha}: E_{\alpha} \rightarrow$ $\mathbb{L}_{X_{\alpha} / S}$ on an étale cover $\left\{X_{\alpha} \rightarrow X\right\}_{\alpha \in A}$ of $X$. Let $\sigma: \mathcal{O} b_{X} \rightarrow \mathcal{O}_{X}$ be a cosection. We follow the notation of Section 4 throughout.

Suppose that we have a Cartesian diagram

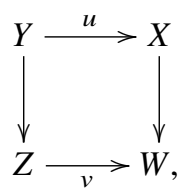

where $Z, W$ are smooth varieties and $v$ is a regular embedding, so that we also have Cartesian diagram

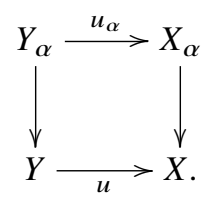


Suppose now that we have an almost perfect obstruction theory on $Y \rightarrow S$ given by perfect obstruction theories $\phi_{\alpha}^{\prime}: E_{\alpha}^{\prime} \rightarrow \mathbb{L}_{Y_{\alpha} / S}$ together with commutative diagrams

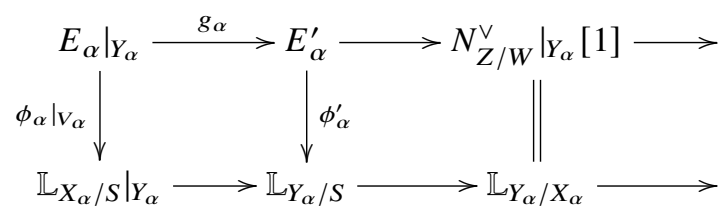

of distinguished triangles that are compatible with the diagrams (2.5) for $\phi$ and $\phi^{\prime}$ such that we have exact sequences

$$
\left.\left.N_{Z / W}\right|_{Y_{\alpha}} \longrightarrow \mathcal{O} b_{Y_{\alpha}} \stackrel{h^{1}\left(g_{\alpha}^{\vee}\right)}{\longrightarrow} \mathcal{O} b_{X_{\alpha}}\right|_{Y_{\alpha}} \longrightarrow 0
$$

that glue to a sequence

$$
\left.\left.N_{Z / W}\right|_{Y} \longrightarrow \mathcal{O} b_{Y} \longrightarrow \mathcal{O} b_{X}\right|_{Y} \longrightarrow 0
$$

We obtain an induced cosection

$$
\sigma^{\prime}:\left.\mathcal{O} b_{Y} \longrightarrow \mathcal{O} b_{X}\right|_{Y} \stackrel{\left.\sigma\right|_{Y}}{\longrightarrow} \mathcal{O}_{Y} .
$$

The Cartesian squares (A.1) and (A.2) give rise to a diagram

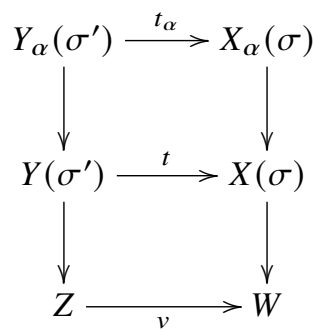

with Cartesian squares.

Theorem A.1. $\left[\mathcal{G}_{Y, \mathrm{loc}}^{\mathrm{vir}}\right]=v^{!}\left[\bigcirc_{X, \mathrm{loc}}^{\mathrm{vir}}\right] \in K_{0}\left(Y\left(\sigma^{\prime}\right)\right)$.

Here the Gysin map $v^{!}: K_{0}(X(\sigma)) \rightarrow K_{0}\left(Y\left(\sigma^{\prime}\right)\right)$ is defined by the formula

$$
v^{!}[\mathcal{A}]=\left[\left.\circlearrowleft_{Z}^{W}\right|_{X(\sigma)} \otimes_{\mathscr{O}_{X(\sigma)}} \mathcal{A}\right] \in K_{0}\left(Y\left(\sigma^{\prime}\right)\right)
$$

where we fix $\circlearrowleft_{Z}^{W}$ to be a finite locally free resolution of $v_{*} \bigcirc_{Z}$. By [19], $v^{!}$also equals the composition

$$
K_{0}(X(\sigma)) \stackrel{\sigma_{u}}{\longrightarrow} K_{0}\left(C_{Y\left(\sigma^{\prime}\right) / X(\sigma)}\right) \stackrel{0_{N_{Z / W}}^{!}}{\longrightarrow} K_{0}\left(Y\left(\sigma^{\prime}\right)\right),
$$

where $\sigma_{u}$ is specialization to the normal cone and $0 !_{N_{Z / W}}$ is the Gysin map induced from the Cartesian diagram

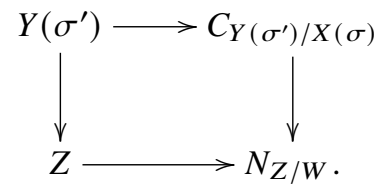


Proof of Theorem A.1. Let $\mathcal{M}_{X}^{\circ} \rightarrow \mathbb{P}^{1}$ be the deformation of $X$ to its intrinsic normal cone stack $\mathcal{C}_{X}$ and $\mathcal{W}=\mathcal{M}_{Y \times \mathbb{P}^{1} / \mathcal{M}_{X}^{\circ}}^{\circ}$ be the double deformation space given by the deformation of $Y \times \mathbb{P}^{1}$ inside $\mathcal{M}_{X}^{\circ}$

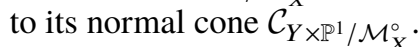

As in the proof of Lemma 5.7, we obtain

$$
\left[\sigma_{\mathcal{C}_{Y}}\right]=\left[\sigma_{\mathcal{C}_{Y / \mathcal{C}_{X}}}\right] \in K_{0}\left(\mathcal{C}_{Y \times \mathbb{P}^{1} / \mathcal{M}_{X}^{\circ}}\right)
$$

Since $\mathcal{C}_{Y \times \mathbb{P}^{1} / \mathcal{M}_{X}^{\circ}}$ is a closed substack of $\mathcal{N}_{Y \times \mathbb{P}^{1} / \mathcal{M}_{X}^{\circ}}$, the equality holds in $K_{0}\left(\mathcal{N}_{Y \times \mathbb{P}^{1} / \mathcal{M}_{X}^{\circ}}\right)$ as well.

Following [17], for each index $\alpha$, we consider the commutative diagram of distinguished triangles on $Y_{\alpha} \times \mathbb{P}^{1}$

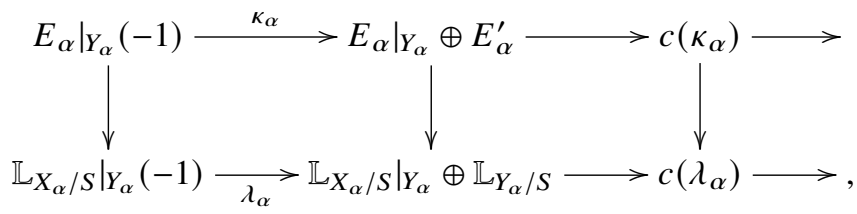

where $\kappa_{\alpha}=\left(T \cdot \mathrm{id}, U \cdot g_{\alpha}\right)$ with $T, U$ coordinates on $\mathbb{P}^{1}$.

Clearly, $\lambda_{\alpha}$ is the restriction to $V_{\alpha}$ of a global morphism $\lambda$. By [17], we have that $h^{1} / h^{0}\left(c(\lambda)^{\vee}\right)=$ $\mathcal{N}_{Y \times \mathbb{P}^{1} / \mathcal{M}_{X}^{\circ}}$.

By the properties of almost perfect obstruction theories and the compatibility diagrams (A.3), the closed embeddings

$$
h^{1}\left(c\left(\lambda_{\alpha}\right)^{\vee}\right) \longrightarrow h^{1}\left(c\left(\kappa_{\alpha}\right)^{\vee}\right)
$$

glue to a closed embedding of sheaf stacks on $Y \times \mathbb{P}^{1}$

$$
\mathfrak{n}_{Y \times \mathbb{P}^{1} / \mathcal{M}_{X}^{\circ}} \longrightarrow \mathcal{K}
$$

The same argument works at the level of coarse moduli sheaves, where flatness stands for exactness of the pullback functor. Thus we deduce the equality

$$
\left[\mathcal{O}_{\mathfrak{c}_{Y}}\right]=\left[\mathcal{O}_{\mathfrak{C}_{Y / \mathcal{C}_{X}}}\right] \in K_{0}(\mathcal{K})
$$

The top row of (A.9) together with (A.4) and (A.5) give a commutative diagram

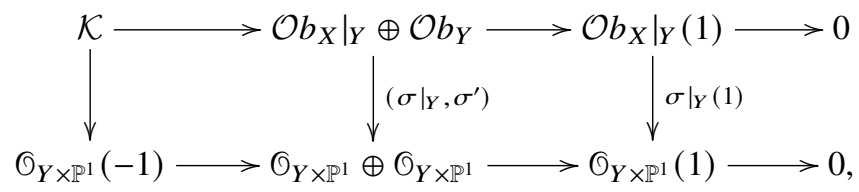

and therefore we obtain a (twisted) cosection $\bar{\sigma}: \mathcal{K} \rightarrow L$, where $L$ is the line bundle $\sigma_{Y \times \mathbb{P}^{1}}(-1)$.

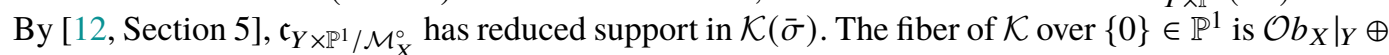
$\left.N_{Z / W}\right|_{Y}$, while the fiber over $\{1\} \in \mathbb{P}^{1}$ is $\mathcal{O} b_{Y}$. The cosection $\bar{\sigma}$ also restricts to the corresponding cosections over these two fibers. Therefore, we obtain by (A.10)

$$
\left[\mathcal{O}_{Y, \mathrm{loc}}^{\mathrm{vir}}\right]=0_{\mathcal{O} b_{Y}, \sigma^{\prime}}\left[\mathcal{O}_{\mathcal{C}_{Y}}\right]=0_{\left.\left.\mathcal{O} b_{X}\right|_{Y} \oplus N_{Z / W}\right|_{Y},\left.\sigma\right|_{Y}}^{!}\left[\mathcal{O}_{\mathfrak{c}_{Y / \mathcal{C}_{X}}}\right]
$$


Now, since the usual properties of Gysin maps hold by working on local charts of the corresponding sheaf stacks, we have

$$
0_{\left.\left.\mathcal{O} b_{X}\right|_{Y} \oplus N_{Z / W}\right|_{Y},\left.\sigma\right|_{Y}}^{!}\left[\mathcal{O}_{\mathfrak{c}_{Y / \mathcal{C}_{X}}}\right]=0 !_{\left.\mathcal{O} b_{X}\right|_{Y},\left.\sigma\right|_{Y}} 0_{\left.N_{Z / W}\right|_{Y}}^{!}\left[\mathcal{G}_{\mathfrak{c}_{Y / \mathcal{C}_{X}}}\right]=0 !{ }_{\left.\mathcal{O} b_{X}\right|_{Y},\left.\sigma\right|_{Y}} v^{!}\left[\mathcal{O}_{\mathfrak{c}_{X}}\right]
$$

By the next proposition, we have $0 !{ }_{\left.\mathcal{O} b_{X}\right|_{Y},\left.\sigma\right|_{Y}} v^{!}=v ! 0_{\mathcal{O} b_{X}, \sigma}$, which implies the desired equality.

Proposition A.2. For any coherent sheaf $\mathcal{A}$ on $\mathcal{O} b_{X}$ supported on $\mathcal{O} b_{X}(\sigma), 0_{\left.\mathcal{O} b_{X}\right|_{Y},\left.\sigma\right|_{Y}}^{!} \nu^{!}[\mathcal{A}]=$ $v ! 0_{\mathcal{O} b_{X}, \sigma}[\mathcal{A}]$.

Proof. Since the pullback $v^{!}$is given by tensoring with the resolution $\sigma_{Z}^{W}$, the equality follows by combining the proofs of [14, Lemma 5.6] and [16, Proposition 4.3] together with the construction of the cosection localized Gysin map in Section 4.

Acknowledgements. Y.-H. Kiem was partially supported by Samsung Science and Technology Foundation grant SSTF-BA160101 .

Conflict of Interest: None.

\section{References}

[1] Jarod Alper, Jack Hall and David Rydh. A Luna étale slice theorem for algebraic stacks. Ann. of Math. (2), 191(3): 675-738, 2020.

[2] K. Behrend. Gromov-Witten invariants in algebraic geometry. Invent. Math., 127(3): 601-617, 1997.

[3] Kai Behrend. Donaldson-Thomas type invariants via microlocal geometry. Ann. of Math. (2), 170(3): 1307-1338, 2009.

[4] Kai Behrend and Barbara Fantechi. The intrinsic normal cone. Invent. Math., 128(1): 45-88, 1997.

[5] Huai-Liang Chang, Young-Hoon Kiem and Jun Li. Torus localization and wall crossing for cosection localized virtual cycles. Adv. Math., 308: 964-986, 2017.

[6] Dan Edidin and William Graham. Nonabelian localization in equivariant $K$-theory and Riemann-Roch for quotients. $A d v$. Math., 198(2): 547-582, 2005.

[7] Tom Graber and Rahul Pandharipande. Localization of virtual classes. Invent. Math., 135(2): 487-518, 1999.

[8] Robin Hartshorne. Algebraic geometry. Springer-Verlag, New York-Heidelberg, 1977. Graduate Texts in Mathematics, No. 52.

[9] Michi-aki Inaba. Toward a definition of moduli of complexes of coherent sheaves on a projective scheme. J. Math. Kyoto Univ., 42(2): 317-329, 2002.

[10] Yunfeng Jiang and Richard P. Thomas. Virtual signed Euler characteristics. J. Algebraic Geom., 26(2): 379-397, 2017.

[11] Young-Hoon Kiem. Localizing virtual fundamental cycles for semi-perfect obstruction theories. Internat. J. Math., 29(4): 1850032, 30, 2018.

[12] Young-Hoon Kiem and Jun Li. Localizing virtual cycles by cosections. J. Amer. Math. Soc., 26(4): 1025-1050, 2013.

[13] Young-Hoon Kiem and Jun Li. A wall crossing formula of Donaldson-Thomas invariants without Chern-Simons functional. Asian J. Math., 17(1): 63-94, 2013.

[14] Young-Hoon Kiem and Jun Li. Localizing virtual structure sheaves by cosections. arXiv e-prints, page arXiv:1705.09458, May 2017.

[15] Young-Hoon Kiem, Jun Li and Michail Savvas. Generalized Donaldson-Thomas Invariants via Kirwan Blowups. arXiv e-prints, page arXiv:1712.02544, December 2017.

[16] Young-Hoon Kiem and Michail Savvas. K-theoretic generalized Donaldson-Thomas invariants. International Mathematics Research Notices, 05 2020. rnaa097.

[17] Bumsig Kim, Andrew Kresch and Tony Pantev. Functoriality in intersection theory and a conjecture of Cox, Katz, and Lee. J. Pure Appl. Algebra, 179(1-2): 127-136, 2003.

[18] Andrew Kresch. Cycle groups for Artin stacks. Invent. Math., 138(3): 495-536, 1999.

[19] Yuan-Pin Lee. Quantum K-theory. I. Foundations. Duke Math. J., 121(3): 389-424, 2004.

[20] Jun Li and Gang Tian. Virtual moduli cycles and Gromov-Witten invariants of algebraic varieties. J. Amer. Math. Soc., 11(1): 119-174, 1998.

[21] Max Lieblich. Moduli of complexes on a proper morphism. J. Algebraic Geom., 15(1): 175-206, 2006.

[22] Cristina Manolache. Virtual pull-backs. J. Algebraic Geom., 21(2): 201-245, 2012.

[23] Andrei Okounkov. Lectures on K-theoretic computations in enumerative geometry. In Geometry of moduli spaces and representation theory, volume 24 of IAS/Park City Math. Ser., pages 251-380. Amer. Math. Soc., Providence, RI, 2017.

[24] Andrei Okounkov. Takagi lectures on Donaldson-Thomas theory. Jpn. J. Math., 14(1): 67-133, 2019. 
[25] Rahul Pandharipande and Richard P. Thomas. Curve counting via stable pairs in the derived category. Invent. Math., 178(2): 407-447, 2009.

[26] Feng Qu. Virtual pullbacks in K-theory. Ann. Inst. Fourier (Grenoble), 68(4): 1609-1641, 2018.

[27] Matthieu Romagny. Group actions on stacks and applications. Michigan Math. J., 53(1): 209-236, 2005.

[28] Michail Savvas. Generalized Donaldson-Thomas Invariants of derived objects via Kirwan blowups. arXiv e-prints, page arXiv:2005.13768, May 2020.

[29] Richard P. Thomas. A holomorphic Casson invariant for Calabi-Yau 3-folds, and bundles on K3 fibrations. J. Differential Geom., 54(2): 367-438, 2000.

[30] Richard P. Thomas. Equivariant $K$-theory and refined Vafa-Witten invariants. Comm. Math. Phys., 378(2): $1451-1500,2020$.

[31] B. Toen. Théorèmes de Riemann-Roch pour les champs de Deligne-Mumford. K-Theory, 18(1): 33-76, 1999. 